\title{
Reconstitution de la dynamique du paysage alluvial de trois secteurs fonctionnels de la rivière allier (1946-2000), Massif central, France \\ Reconstitution of the dynamics of the alluvial landscape of three functional sectors of the Allier River (1946-2000), Central Massif, France.
}

\author{
Stéphane Petit
}

Volume 60, numéro 3, 2006

URI : https://id.erudit.org/iderudit/018000ar

DOI : https://doi.org/10.7202/018000ar

Aller au sommaire du numéro

Éditeur(s)

Les Presses de l'Université de Montréal

ISSN

0705-7199 (imprimé)

1492-143X (numérique)

Découvrir la revue

Citer cet article

Petit, S. (2006). Reconstitution de la dynamique du paysage alluvial de trois secteurs fonctionnels de la rivière allier (1946-2000), Massif central, France. Géographie physique et Quaternaire, 60(3), 271-287.

https://doi.org/10.7202/018000ar

\section{Résumé de l'article}

L'écocomplexe de la plaine d'inondation de la rivière Allier a été étudié dans trois secteurs fonctionnels présentant une dynamique géomorphologique contrastée : le secteur à méandres de Joze-Maringues où l'Allier est corseté par des enrochements et touché par les extractions de granulats, le secteur à méandres mobiles de la Réserve Naturelle du Val d'Allier et le secteur à bras multiples situé entre les villages de Villeneuve-sur-Allier et du Veurdre. L'étude se fonde sur six à huit missions de photographies aériennes prises entre 1946 et 2000 pour déceler la dynamique du paysage alluvial. L'occupation du sol, la largeur de la bande active et le taux d'érosion des berges ont été estimés par photo-interprétation et leur évolution spatiotemporelle a été mise en évidence par des traitements statistiques et cartographiques. Les résultats indiquent un accroissement des surfaces vouées à l'agriculture à partir des années 1950 et une progression du recouvrement de la végétation arborée en lien avec la modification des pratiques culturales et la rétroaction de la bande active. Dans les trois secteurs, le pourcentage de la végétation arborée est passé de 10 à $17 \%$ en 50 ans. Depuis la seconde moitié du $20^{\mathrm{e}}$ siècle, la modification des pratiques agro-pastorales semble être un agent prépondérant pour expliquer la végétalisation progressive de la plaine alluviale de la rivière Allier. L'érosion des berges ne s'est pas atténuée durant cette période. Elle était plus active pendant la décennie 1970-1980 en raison des crues plus fréquentes et plus intenses.
Tous droits réservés (C) Les Presses de l’Université de Montréal, 2008
Ce document est protégé par la loi sur le droit d'auteur. L’utilisation des services d’Érudit (y compris la reproduction) est assujettie à sa politique d'utilisation que vous pouvez consulter en ligne.

https://apropos.erudit.org/fr/usagers/politique-dutilisation/ 


\section{RECONSTITUTION DE LA DYNAMIQUE DU PAYSAGE ALLUVIAL DE TROIS SECTEURS FONCTIONNELS DE LA RIVIĖRE ALLIER (1946-2000), MASSIF CENTRAL, FRANCE}

Stéphane PETIT*, Laboratoire de géographie physique et environnementale GEOLAB, Université Blaise Pascal, UMR 6042 CNRS, 4 rue Ledru, 63057 Clermont-Ferrand Cedex, France.

RÉSUMÉ L'écocomplexe de la plaine d'inondation de la rivière Allier a été étudié dans trois secteurs fonctionnels présentant une dynamique géomorphologique contrastée: le secteur à méandres de JozeMaringues où l'Allier est corseté par des enrochements et touché par les extractions de granulats, le secteur à méandres mobiles de la Réserve Naturelle du Val d'Allier et le secteur à bras multiples situé entre les villages de Villeneuve-sur-Allier et du Veurdre. L'étude se fonde sur six à huit missions de photographies aériennes prises entre 1946 et 2000 pour déceler la dynamique du paysage alluvial. L'occupation du sol, la largeur de la bande active et le taux d'érosion des berges ont été estimés par photo-interprétation et leur évolution spatiotemporelle a été mise en évidence par des traitements statistiques et cartographiques. Les résultats indiquent un accroissement des surfaces vouées à l'agriculture à partir des années 1950 et une progression du recouvrement de la végétation arborée en lien avec la modification des pratiques culturales et la rétroaction de la bande active. Dans les trois secteurs, le pourcentage de la végétation arborée est passé de 10 à $17 \%$ en 50 ans. Depuis la seconde moitié du $20^{\circ}$ siècle, la modification des pratiques agro-pastorales semble être un agent prépondérant pour expliquer la végétalisation progressive de la plaine alluviale de la rivière Allier. L'érosion des berges ne s'est pas atténuée durant cette période. Elle était plus active pendant la décennie 1970-1980 en raison des crues plus fréquentes et plus intenses.
ABSTRACT Reconstitution of the dynamics of the alluvial landscape of three functional sectors of the Allier River (1946-2000), Central Massif, France. The landscape mosaics of the Allier river floodplain has been studied in three functional reaches with contrasted geomorphic activities: the Joze-Maringues meander reach where the river is constrained by embankments and affected by gravel mining, the shifting meander reach of the Val d'Allier Natural Reserve, and the anabranching reach between the village of Villeneuve-sur-Allier and Le Veurdre. This study is based on six to eight aerial photography, series taken between 1946 and 2000, to underline the dynamics of the alluvial landscape. Land cover types, active channel width and river bank erosion rate have been assessed from the photo interpretation, and their change in space and time has been statistically analysed and mapped. The results point to an increase of the cultivated areas from the 1950's, and an extension of the tree-cover in relation with a change of agricultural practices and the decrease of the channel activity. In the three reaches, the percentage of the tree-cover has moved from 10 to $17 \%$ in 50 years. From the second half of the 20th century, the change in the agro-pastoral practices seems to be a major factor explaining the increase of the floodplain tree and bush cover. Bank erosion has not diminished during this period. It was more active in the 1970-1980 decade owing to more severe and frequent floods. 


\section{INTRODUCTION}

Les plaines d'inondation des grands cours d'eau sont composées d'une mosaïque d'unités écologiques instables (Pautou et Décamps, 1985; Bravard et al., 1986; Amoros et al., 1987) dont la richesse des habitats et des espèces associées est produite et maintenue par l'hétérogénéité spatiale et temporelle des processus géomorphologiques (Brinson et al., 1981; Gregory et al., 1991; Malanson, 1993; Shankman, 1993; Hughes, 1994; Ward et al., 1999; Ward et Wiens, 2001). Le régime hydrologique est un facteur clé influençant les chenaux, les plaines d'inondation et la végétation des plaines d'inondation (Hupp et Osterkamp, 1985; Salo et al., 1986; Junk et al., 1989; Naiman et al., 1993; Hughes, 1997; Bendix et Hupp, 2000), où l'eau joue un rôle essentiel dans l'organisation et l'évolution des paysages alluviaux (Naiman, 1996). Les processus fluviaux - tels que les fluctuations de débits définies par les concepts de flood pulse et de flow pulse (Junk et al., 1989 ; Puckridge et al., 1998), le recoupement des méandres, l'instabilité latérale du chenal, l'érosion et le dépôt d'alluvions - structurent la plaine d'inondation et créent une mosaïque de milieux variant dans l'espace et le temps. Ces perturbations hydrologiques et géomorphologiques influencent de manière significative la position des différentes unités écologiques par rapport au chenal actif et leur développement sur la plaine d'inondation.

Les recherches menées depuis une vingtaine d'années en géomorphologie fluviale et en écologie du paysage alluvial ont montré que les corridors fluviaux subissent une métamorphose progressive. Les actions anthropiques, et indirectement leurs impacts, telle que l'incision du chenal (Bravard et al., 1997), influencent de nombreux processus dont les processus hydrologiques, sédimentologiques et biotiques qui façonnent les paysages alluviaux.

Au cours de la seconde moitié du $20^{\mathrm{e}}$ siècle, dans les plaines d'inondation des pays occidentaux, la création de barrages, la mise en place de digues et d'enrochements, ainsi que les extractions de granulats dans le chenal principal font partie des activités humaines dominantes qui ont affecté la dynamique de la rivière, le régime hydrologique et son transport solide. Depuis les cinquante dernières années, une abondante littérature fait état de ces perturbations anthropiques et des impacts qu'elles ont eu sur l'écocomplexe alluvial en Europe (Pautou, 1984; Pautou et al., 1992; Décamps, 1993; Hughes, 1994 ; Piégay, 1995 ; Bravard et al., 1997; Surian, 1999 ; Piégay et al., 2003a; Steiger et al., 2005), aux ÉtatsUnis (Johnson, 1994; Gurnell et Gregory, 1995; Miller et al., 1995 ; Johnson, 1998; Merritt et Cooper, 2000 ; Cooper et al., 2003) et en Australie (Brierley et al., 1999; Kingsford, 2000; Gehrke et Harris, 2001). Ces impacts anthropiques se traduisent le plus souvent par la suppression de la dynamique naturelle (Peiry, 1997), la simplification de la morphologie du chenal (Sidle et Sharma, 1986 ; Steiger et al., 2000; Pisùt, 2002), l'isolement du chenal principal de sa plaine d'inondation par diminution de la largeur du lit (Eschner et al., 1983) et le changement de physionomie de la plaine d'inondation (Johnson et al., 1992; Johnson, 1997). Ces interventions ont très largement contribué à modifier la dynamique des paysages allu- viaux et leurs conséquences restent encore difficiles à prédire (Naiman, 1992).

En France, des recherches menées sur les grands cours d'eau, comme le Rhône (Bravard, 1985; Pautou et Décamps, 1985), la Garonne (Steiger et al., 1998) et l'Ain (Marston et al., 1995), montrent que la simplification de ces derniers au cours du temps est une constante et la progression de la végétation rivulaire est l'une des principales tendances de la dynamique paysagère. Des rivières de piémont (Isère, Drac, Romanche), de grands cours d'eau (Ain, Loire, Garonne, Rhône) ainsi que la plupart des rivières du sud-est de la France (Gautier, 1992; Piégay et al., 1994 ; Liébault et Piégay, 2002) sont touchés par cette végétalisation dont la temporalité et les mécanismes d'implantation sont différents. Deux principaux types d'invasion de la végétation ont été observés par Piégay et al. (2003b). Ces auteurs expliquent que, au début du $20^{\mathrm{e}}$ siècle, l'invasion de la végétation a touché les secteurs tressés des rivières de montagne (par exemple l'Ubaye dans les années 1920) en réponse à la diminution de la charge sédimentaire entraînée par la reforestation du bassin versant et l'implantation d'ouvrages de rétention sédimentaire entre 1880 et 1910; le développement spontané de la végétation a été enregistré entre 1945 et 1970 sur l'Ain, mais également sur d'autres cours d'eau (Eygues, Roubion, Drôme, Ouvèze, Loire, Allier) où l'écoulement n'est pas influencé par les barrages. Cette progression des boisements est souvent associée à une rétraction de la bande active (Dufour, 2005) et bien souvent expliquée par la modification de facteurs de contrôle amont que sont la réduction du transport solide et du régime de crue. Or, à partir d'observations effectuées sur les cours d'eau du bassin rhodanien (tabl. I), Liébault et Piégay (2002) expliquent que la rétraction de la bande active et l'augmentation des surfaces de boisements peuvent également être expliqués par un contrôle local, à savoir la modification des pratiques riveraines en lit majeur (activités pastorales, coupes de bois) et l'incision du lit en raison des extractions.

L'objectif de cet article est de préciser la dynamique géomorphologique et le fonctionnement contemporain (19462000) de la plaine alluviale de l'Allier inférieur et de mieux comprendre les mécanismes d'évolution de son paysage fluvial en relation avec les activités humaines et les changements naturels. Puis, il est de tester l'hypothèse selon laquelle les changements paysagers relèvent d'un contrôle anthropique local et d'un ajustement des conditions morphologiques locales. En dernier lieu, il s'agit d'évaluer si les processus géomorphologiques et socio-économiques contrôlent la structuration de la plaine d'inondation.

Afin d'identifier les causes et les facteurs clés responsables des changements du paysage alluvial, cette analyse est fondée sur une approche quantitative des changements structurels de la plaine d'inondation (Marston et al., 1995; Mendonça-Santos, 1999; Cousins, 2001 ; Mendonça-Santos et Claramunt, 2001). L'étude s'appuie sur une analyse diachronique à l'échelle de trois secteurs fonctionnels (Amoros et al., 1988) à fonctionnement géomorphologique et d'impact anthropique différents. La compréhension des changements intègre à la fois les facteurs naturels et l'historique des activités humaines. L'intérêt d'une telle approche s'appuie sur la 
TABLEAU I

Causes de la rétraction des bandes actives de l'Allier (Liébault et Piégay, 2002)

\begin{tabular}{|c|c|c|c|}
\hline Influence & Facteurs & Causes & Remarques \\
\hline \multirow[t]{8}{*}{ Amont } & $\begin{array}{l}\text { Réduction du transport } \\
\text { solide }\end{array}$ & $\begin{array}{l}\text { Fin du Petit Age Glaciaire } \\
(1850-1880)\end{array}$ & $\begin{array}{l}\text { Réduction des apports antérieure à la rétraction } \\
\text { (temps de réponse) }\end{array}$ \\
\hline & & Construction de barrages & Rétraction progressive vers l'aval depuis les \\
\hline & & $\begin{array}{l}\text { Végétalisation du bassin versant } \\
\text { (spontanée ou plantations) }\end{array}$ & sources sédimentaires \\
\hline & & Correction torrentielle & \\
\hline & $\begin{array}{l}\text { Réduction du régime } \\
\text { de crue }\end{array}$ & $\begin{array}{l}\text { Fin du Petit Age Glaciaire } \\
(1850-1880)\end{array}$ & Végétalisation et rétraction synchrones \\
\hline & & $\begin{array}{l}\text { Période hydrologique particulière } \\
\text { durant le } 20^{\circ} \text { siècle }\end{array}$ & \\
\hline & & Construction de barrages & \\
\hline & & $\begin{array}{l}\text { Végétalisation du bassin versant } \\
\text { (spontanée ou plantations) }\end{array}$ & \\
\hline \multirow[t]{2}{*}{ Locale } & Incision du chenal & $\begin{array}{l}\text { Extraction et dégraissement des } \\
\text { bancs }\end{array}$ & $\begin{array}{l}\text { Incision antérieure à la végétalisation du lit mineur } \\
\text { Rétraction progressive depuis le site d'extraction }\end{array}$ \\
\hline & $\begin{array}{l}\text { Végétalisation de } \\
\text { la plaine alluviale }\end{array}$ & Modification des pratiques pastorales & Végétalisation et rétraction synchrones \\
\hline
\end{tabular}

comparaison de secteurs représentatifs de la plaine alluviale de l'Allier présentant des dynamiques fluviales et des interventions humaines contrastées. L'observation de secteurs morphologiquement différents appartenant à divers degrés d'anthropisation est nécessaire afin de distinguer la trajectoire évolutive du paysage alluvial des ajustements locaux, et d'évaluer si les conditions locales influencent de la même manière l'évolution de la plaine d'inondation.

\section{SITES D'ÉTUDE}

De sa source (1 $485 \mathrm{~m}$ ) à la confluence avec la Loire (Bec d'Allier, $167 \mathrm{~m}$ ), l'Allier parcourt $410 \mathrm{~km}$, draine un bassin versant de $14400 \mathrm{~km}^{2}$ et atteint à Moulins un module de $141 \mathrm{~m}^{3} / \mathrm{s}$ (fig. 1A, tabl. II). Les sites étudiés se situent exclusivement sur l'Allier inférieur en aval de Brioude (fig. 1B). La délimitation longitudinale des secteurs d'étude provient de la sectorisation en tronçons et sous-tronçons définie par la DIREN Auvergne-Epteau (1998). Elle a été effectuée sur la base de critères géologiques, géomorphologiques, hydrologiques et anthropiques. Cette étude couplée à d'autres documents (Centre d'Études Techniques de l'Équipement de Lyon, 1982; Luherne, 1994 ; Dejaifve et Pinoche, 1998; Saillard, 1998, 1999 ; Conservatoire des Espaces et des Paysages d'Auvergne, 2001) a servi de référence dans le choix des sites à fonctionnement morphologiquement contrasté et dans la présélection de zones potentiellement intéressantes. Ces dernières ont fait l'objet d'une analyse diachronique de l'évolution du chenal actif pour diagnostiquer ses potentialités de divagation. Le résultat a conduit à la sélection de trois secteurs à fonctionnement morphologique homogène et représentatifs de l'Allier inférieur: (1) le secteur à méandres de JozeMaringues (fig. 1C), (2) le secteur à méandres mobiles de la Réserve Naturelle du Val d'Allier (fig. 1D), et (3) le secteur à bras multiples et relativement stables situé entre les villages de Villeneuve-sur-Allier et du Veurdre (fig. 1E). Ce choix se justifie par leurs aptitudes différentes au rajeunissement des habitats et l'histoire contrastée des interventions humaines depuis les cinquante dernières années.

\section{SECTEUR DE JOZE-MARINGUES}

Le secteur de Joze-Maringues (secteur amont; fig. 1C, tabl. II) se caractérise par un style fluvial sinueux proche du méandrage. Dès le début des années 1970, la plaine d'inondation a subi une exploitation intensive de ses ressources alluvionnaires, nécessitant localement la rectification du tracé du chenal. Aujourd'hui, la plupart des sinuosités sont protégées par des enrochements qui représentent une longueur totale de $5,3 \mathrm{~km}$, soit $24 \%$ des berges, les deux rives étant incluses. Depuis 1982, l'extraction des granulats dans le lit mineur de l'Allier est interdite; elle se poursuit désormais dans le lit majeur où une forêt alluviale reste encore largement développée.

\section{SECTEUR DE CHÂTEL-DE-NEUVRE}

Le secteur de Châtel-de-Neuvre (secteur intermédiaire; fig. 1D, tabl. II) se situe dans la Réserve Naturelle du Val d'Allier, entre les ponts de Châtel-de-Neuvre et la route nationale 14. Aujourd'hui, le chenal affiche un style méandriforme mais le tressage, présent jusque dans les années 1950, tend à réapparaître localement depuis une dizaine d'années. Ce site, déclaré Réserve Naturelle en 1994, constitue un des rares témoins en Europe occidentale, avec la Dvina du nord, l'Oder et la Vistule, d'un système fluvial de plaine encore peu altéré par l'homme (Décamps, 1991 cité dans Dejaifve et Pinoche, 1998). Le fonctionnement de l'Allier y est considéré 


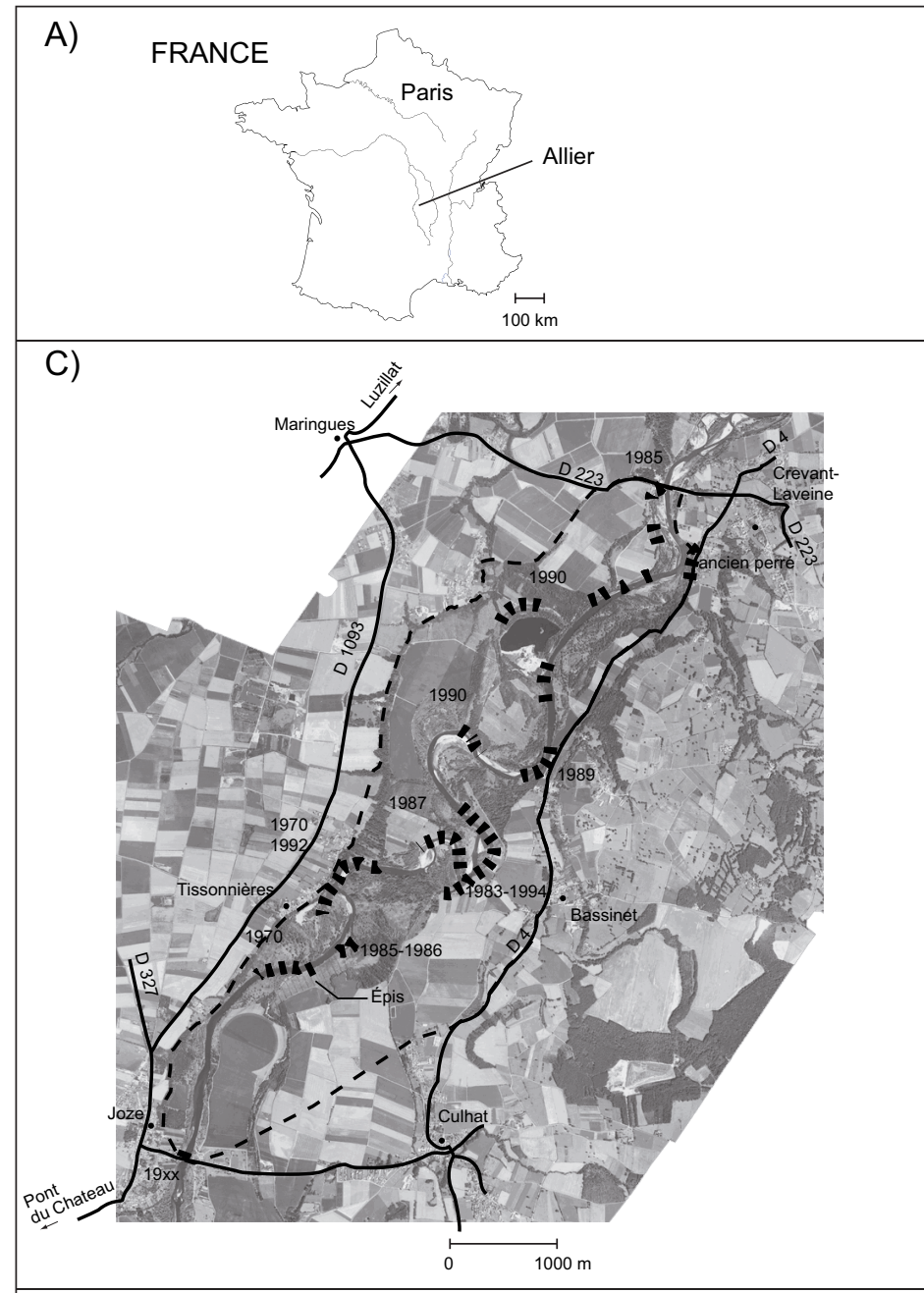

B)

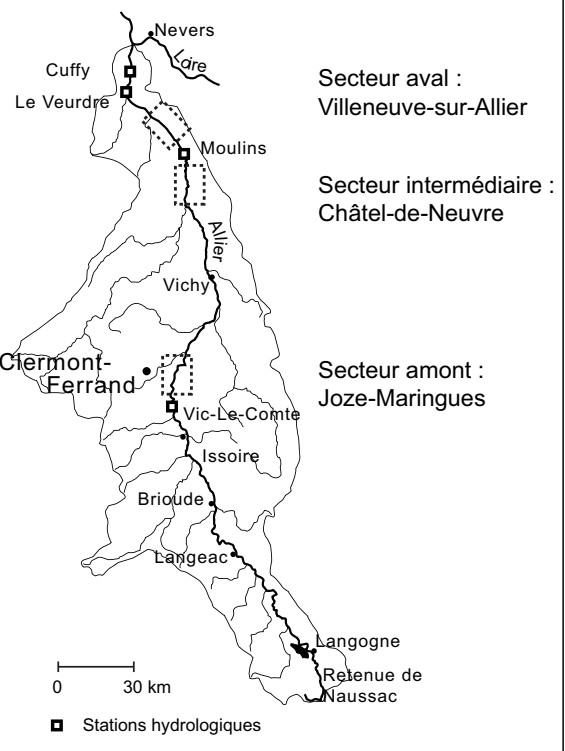

D)

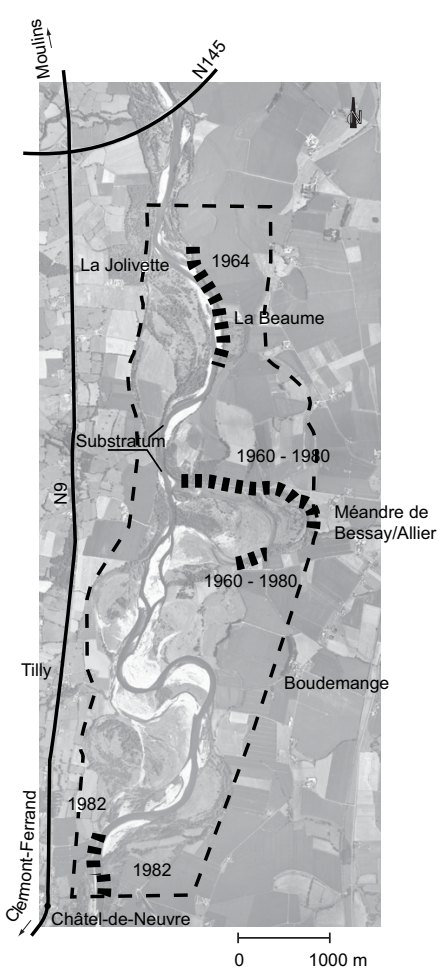

_ _ Fuseau de mobilité (cf. Malavoi, 1998)

III Enrochements

1960 Date de construction

- Principales routes

Source des photographies (A), (B) et (C) :

Mosaique aerienne issue de la campagne de prise de

vervice de Bassin Loire-Bretagne - Direction Régionale de l'Environnement.
FIGURE 1. Sites d'étude sur la rivière Allier: (A) localisation de l'Allier à l'échelle nationale, (B) localisation des sites d'étude à l'échelle du bassin versant, (C) secteur de Joze-Maringues, (D) secteur de Châtelde-Neuvre et (E) secteur de Villeneuve-sur-Allier.
Study sites on the Allier River: (A) location of the Allier at the national scale, $(B)$ location of study sites at the watershed scale, $(C)$ reach of Joze-Maringues, $(D)$ reach of Châtel-de-Neuvre and $(E)$ reach of Villeneuve-sur-Allier. 
TABLEAU II

Principales caractéristiques de la rivière Allier au droit des secteurs de Joze-Maringues, Châtel-de-Neuvre et Villeneuve-sur-Allier

\begin{tabular}{|c|c|c|c|c|}
\hline Caractéristique & Unité & $\begin{array}{c}\text { Joze-Maringues } \\
\text { Station hydrologique } \\
\text { Vic-le-Comte } \\
(1933-2005)\end{array}$ & $\begin{array}{c}\text { Châtel-de-Neuvre } \\
\text { Station hydrologique } \\
\text { Moulins } \\
(1968-2005)\end{array}$ & $\begin{array}{c}\text { Villeneuve-sur-Allier } \\
\text { Station hydrologique } \\
\text { Cuffy } \\
(1955-2005)\end{array}$ \\
\hline Superficie des secteurs & ha & 1102 & 1211 & 2099 \\
\hline Longueur du tronçon & $\mathrm{km}$ & 9 & 8,5 & 11,5 \\
\hline Pente & $\mathrm{m} / \mathrm{m}$ & 0,00113 & 0,00064 & 0,00056 \\
\hline Sinuosité & & 1,42 & 1,32 & 1,02 \\
\hline Superficie du bassin versant & $\mathrm{km}^{2}$ & 5370 & 12980 & 14310 \\
\hline Module & $\mathrm{m}^{3} / \mathrm{s}$ & 64,10 & 141 & 150 \\
\hline Crue biennale & $\mathrm{m}^{3} / \mathrm{s}$ & 420 & 720 & 650 \\
\hline Crue décennale & $\mathrm{m}^{3} / \mathrm{s}$ & 700 & 1200 & 1100 \\
\hline Crue vicennale & $\mathrm{m}^{3} / \mathrm{s}$ & 810 & 1400 & 1200 \\
\hline Crue cinquantennale & $\mathrm{m}^{3} / \mathrm{s}$ & 950 & 1700 & 1400 \\
\hline
\end{tabular}

Source: banque HYDRO pour les données hydrologiques.

comme «proche du naturel hormis l'enfoncement du lit lié aux extractions » (DIREN Auvergne-Epteau, 1998). La dynamique latérale est très active, et la géométrie du lit et la taille du chenal sont relativement libres de s'ajuster aux débits et au transport des sédiments. Ce secteur peut être subdivisé en deux parties ayant un fonctionnement géomorphologique contrasté et situées de part et d'autre du méandre de Bessay-sur-Allier. À l'amont de ce méandre, le chenal est latéralement très instable et peut se déplacer de plusieurs dizaines de mètres par an. À l'aval, la rivière est moins mobile du fait de la présence d'enrochements, de terrasses holocènes et, localement, de l'affleurement du substratum marneux. Ce secteur peut être néanmoins traité comme un tronçon homogène puisque la zone aval occupe une plus faible proportion par rapport à la zone amont dont le fonctionnement est représentatif de la majorité du fonctionnement de la Réserve Naturelle.

Le méandre de Bessay-sur-Allier a subi deux recoupements successifs en 1980 et en 1994. Le premier fut un recoupement partiel et correspond à une rectification du tracé effectuée par les services de l'État pour protéger la rive concave de l'érosion. Le second est un recoupement total provoqué par la crue de novembre 1994 dont le débit instantané a atteint $1030 \mathrm{~m}^{3} / \mathrm{s}$ à la station hydrologique de Moulins (fig. 1B). Aujourd'hui, l'intégralité des écoulements se concentre dans un nouveau chenal étroit et rectiligne.

\section{SECTEUR DE VILLENEUVE-SUR-ALLIER}

Localisé dans le bocage bourbonnais, le secteur de Villeneuve-sur-Allier (secteur aval; fig. 1E, tabl. II) affiche, selon la définition de Nanson et Knighton (1996), un style fluvial caractérisé par la présence de chenaux multiples séparés par des îles à végétation semi permanente (anabranching type II). Contrairement à la définition de Nanson et Knighton, le chenal est caractérisé par une faible mobilité latérale et cette configuration morphologique se rapproche de celle décrite sur la Loire moyenne en aval de Tours (Rodriguez, 2004) et dans le secteur de Bréhémont (Grivel et Gautier, 2000). Cette portion ne comporte pratiquement aucune contrainte empêchant la divagation latérale du chenal.

\section{MÉTHODOLOGIE}

\section{INFORMATION SPATIALE}

L'analyse des changements du paysage alluvial de l'Allier s'appuie sur l'utilisation d'une série de photographies aériennes prises entre 1946 et 2000 (tabl. III). Pour toutes les missions, chaque cliché a été rectifié géométriquement et référencé, à l'aide du logiciel ErMapper, afin d'obtenir des images corrigées de la plupart des déformations inhérentes à la prise de vue et aux distorsions causées par l'environnement. L'erreur moyenne quadratique (RMS) est de l'ordre de $3 \mathrm{~m}$. Sur chaque mosaïque de photographies aériennes, 11 unités paysagères ont été digitalisées sur la base de critères visuels, en particulier les critères de reconnaissance développés par Girel (1986), de vérifications sur le terrain (tabl. IV) et selon les séquences typiques de succession végétale des plaines d'inondation européennes (Carbiener, 1970; Pautou, 1984 ; Décamps et al., 1988). La superficie minimale digitalisée est de $4 \mathrm{~m}^{2}$ et la limite latérale des secteurs est le fuseau de mobilité (fig. 1) défini ici comme étant l'espace à préserver pour permettre au cours d'eau de conserver son potentiel d'ajustement en plan et en long. Il est déterminé selon des calculs effectués sur l'amplitude des sinuosités actuelles; les largeurs limites sont adaptées en fonction des contraintes socio-économiques (zones urbanisées, infrastructures routières) ainsi que l'intégration d'autres types de contraintes comme les zones d'extractions, la proximité de zones d'incision et les affleurements marneux. 
TABLEAU III

Missions aériennes utilisées pour l'analyse paysagère des secteurs de Joze-Maringues, Châtel-de-Neuvre et Villeneuve-sur-Allier

\begin{tabular}{|c|c|c|c|c|c|}
\hline Secteur & Année & Mission & Émulsion ${ }^{\mathrm{a}}$ & Échelle & $\begin{array}{l}\text { Débit } \\
\left(\mathrm{m}^{3} / \mathrm{s}\right)\end{array}$ \\
\hline & 1946 & F2430-2630 & $P, N B$ & $1 / 25000$ & étiage \\
\hline & 1954 & F2430-2630 & $\mathrm{P}, \mathrm{NB}$ & $1 / 25000$ & étiage \\
\hline & 1971 & CDP7355 & $P, N B$ & $1 / 20000$ & étiage \\
\hline \multirow[t]{6}{*}{ Joze-Maringues $^{\text {a }}$} & 1985 & IFN6303 & $P, N B$ & $1 / 17000$ & 16,8 \\
\hline & $1992-1993$ & F2631/FD03P & $P, N B$ & $1 / 30000$ & 12 et 37,1 \\
\hline & 2000 & DIREN Centre & $\mathrm{P}, \mathrm{C}$ & $1 / 25000$ & 19 et 17,4 \\
\hline & 1946 & F2627-2728 & $\mathrm{P}, \mathrm{NB}$ & $1 / 25000$ & étiage \\
\hline & 1954 & F2626-2629 & $P, N B$ & $1 / 25000$ & étiage \\
\hline & 1968 & F2528-2628 & $P, N B$ & $1 / 25000$ & 74 \\
\hline \multirow[t]{9}{*}{ Châtel-de-Neuvre b } & 1975 & FR2706 & $P, N B$ & $1 / 15000$ & 40,7 \\
\hline & 1985 & IFN03-63 & IF, C & $1 / 17000$ & 61,5 \\
\hline & 1992 & FD03C & $P, N B$ & $1 / 30000$ & 92 \\
\hline & 1997 & 03IFN97/09 & $P, N B$ & $1 / 20000$ & 52 \\
\hline & 2000 & DIREN Centre & $\mathrm{P}, \mathrm{C}$ & $1 / 25000$ & 50 et 64,5 \\
\hline & 1949 & F2526-2626 & $\mathrm{P}, \mathrm{NB}$ & $1 / 25000$ & étiage \\
\hline & & F2525-2725 & & & \\
\hline & 1957 & F2526-2626 & $P, N B$ & $1 / 25000$ & 250 \\
\hline & 1963 & F2526-2926 & $P, N B$ & $1 / 30000$ & 81 \\
\hline \multirow{4}{*}{ Villeneuve-sur-Allier ${ }^{\mathrm{C}}$} & 1971 & F2526-2626 & $P, N B$ & $1 / 30000$ & étiage \\
\hline & 1985 & IFN03-63 & $P, N B$ & $1 / 17000$ & 50 et 53,5 \\
\hline & 1992 & FD03C & $\mathrm{P}, \mathrm{C}$ & $1 / 30000$ & 92 \\
\hline & 2000 & DIREN Centre & $\mathrm{P}, \mathrm{C}$ & $1 / 25000$ & 50 et 64,5 \\
\hline
\end{tabular}

a $\mathrm{P}=$ panchromatique, $\mathrm{NB}=$ noir et blanc, $\mathrm{IR}=$ infrarouge, $\mathrm{C}=$ couleur.

${ }^{b}$ Débits mesurés à la station hydrométrique de Vic-le-Comte.

$\mathrm{c}, \mathrm{d}$ Débits mesurés à la station hydrométrique de Moulins.

Source: DIREN Centre/banque HYDRO.

TABLEAU IV

Les différentes unités paysagères digitalisées sur les secteurs de Joze-Maringues, Châtel-de-Neuvre et Villeneuve-sur-Allier

\begin{tabular}{|c|c|c|}
\hline Unité & Description & $\begin{array}{l}\text { Critères de différenciation } \\
\text { (Girel, 1986) }\end{array}$ \\
\hline \multirow[t]{2}{*}{ Bande active (eau et bancs nus) } & $\begin{array}{l}\text { Chenal principal et bras morts avec plus ou moins de } \\
\text { végétation aquatique }\end{array}$ & Sombre et lisse \\
\hline & Graviers, sables et limons & Blanc \\
\hline Végétation herbacée & $\begin{array}{l}\text { Végétation herbacée pionnière } \\
\text { Landes, friches et pelouses } \\
\text { Prairie pâturée du lit mineur }\end{array}$ & $\begin{array}{l}\text { Fond blanc (galet), tâches grises éparses, } \\
\text { surfaces grises rugueuses pour les prairies } \\
\text { pâturées }\end{array}$ \\
\hline Végétation arbustive & $\begin{array}{l}\text { Composée majoritairement de Salix sp. (saule pourpre, } \\
\text { argenté et blanc) Populus sp. (Populus nigra) et Alnus } \\
\text { glutinosa et des formations à Prunetalia spinosae (Prunus } \\
\text { spinosae, Crataegus monogyna, Rosa canina) }\end{array}$ & $\begin{array}{l}\text { Fond gris foncé, homogène, houppiers non } \\
\text { individualisés, structure petite et éparse } \\
\text { lorsque houppier seul }\end{array}$ \\
\hline Végétation arborée & $\begin{array}{l}\text { Essentiellement à bois tendres (Salix sp. et Populus sp.) } \\
\text { avec quelques bois durs }\end{array}$ & $\begin{array}{l}\text { Texture rugueuse à cause de la forme des } \\
\text { houppiers individualisés }\end{array}$ \\
\hline \multirow[t]{2}{*}{ Pâturages et cultures } & Prairies grasses & Fond gris foncé \\
\hline & Cultures intensives de blé et maïs & $\begin{array}{l}\text { Parcelle de forme géométrique, présence de } \\
\text { rampes d'irrigation }\end{array}$ \\
\hline Haies & Fraxinus sp., Quercus sp., formation à Prunetalia spinosae & Structure linéaire \\
\hline Habitats & $\begin{array}{l}\text { Maisons d'habitation } \\
\text { Constructions agricoles } \\
\text { Cours et jardins }\end{array}$ & Variables \\
\hline Routes & $\begin{array}{l}\text { Nationales, départementales et chemins d'accès aux } \\
\text { exploitations et champs }\end{array}$ & Structure linéaire bien individualisée, claire \\
\hline Plantations & Majoritairement composées de peupliers hydrides & Houppiers alignés \\
\hline Gravières & En activité ou non & Variables \\
\hline
\end{tabular}


Les cartes vectorielles issues de la digitalisation des unités paysagères sont ensuite transformées en format raster, puis l'évolution du paysage est analysée à partir du Système d'Information Géographique (SIG) Idrisi32 (Eastman, 1997).

\section{DÉTECTION DES CHANGEMENTS DU PAYSAGE}

À partir des cartes raster et grâce aux fonctionnalités du SIG Idrisi32, la détection de l'évolution du paysage est réalisée en utilisant une technique qualitative et quantitative d'analyse des changements, appliquée à des paires de cartes d'occupation du sol successives. Deux types de résultats sont obtenus. Dans un premier temps, des tables de contingence sont créées dans lesquelles les catégories d'une première image sont comparées avec celles d'une seconde image selon le pourcentage de recouvrement de chaque unité. L'analyse des matrices de transition permet de quantifier la dynamique des unités paysagères et, à la manière de Pontius et al. (2004), les proportions de perte et de gain d'une catégorie donnée durant l'intervalle de temps étudié sont calculées. Ces valeurs autorisent alors le calcul des changements totaux (valeur absolue de la différence entre les gains et les pertes) ayant affecté les catégories du paysage. Cependant, comme le soulignent Pontius et al. (2004), il est possible qu'entre les temps 1 et 2 des changements spatiaux aient eu lieu bien que les quantités restent les mêmes; c'est pourquoi des taux d'échanges entre les unités paysagères ont été calculés dans le but de déceler la stabilité de leur position. Dans un second temps, une nouvelle image indiquant la localisation des nouvelles combinaisons d'unités paysagères est produite.

\section{QUANTIFICATION DE LA DYNAMIQUE DU CHENAL}

L'intérêt de l'exploitation des photographies aériennes ne réside pas seulement sur l'obtention d'un instantané du paysage à des dates successives, mais également sur la possibilité qu'elles offrent de calculer des indices quantitatifs décrivant la morphologie en plan du chenal et son activité latérale. Ainsi, la largeur de la bande active et les taux d'érosion ont été mesurés pour les trois secteurs.

La bande active correspond à l'emprise des chenaux en eau et des bancs de galets, de graviers et de sables à l'exclusion des îles végétalisées; l'absence de végétation sur les bancs indique le remaniement des formes fluviales à une fréquence annuelle (Peiry, 1997). Son estimation constitue donc un témoin de la dynamique du cours d'eau et de l'importance du transit de la charge de fond (Rundle, 1985; Peiry et al., 1993). Elle a été calculée sous Maplnfo7 et sa quantification a été effectuée le long de transects équidistants de $100 \mathrm{~m}$ établis perpendiculairement à l'axe d'écoulement. Les données ont été analysées à l'aide du logiciel de statistiques SPSS10.

Les taux d'érosion ont été calculés à partir des cartes d'occupation du sol et grâce aux capacités de superposition d'images et de reclassement qu'offre le logiciel Idrisi32, puis analysés avec le logiciel SPSS10.

\section{RÉSULTATS}

\section{DYNAMIQUE DE L'OCCUPATION DE L'ESPACE PAR LA VÉGÉTATION ARBORÉE}

L'analyse met en évidence une augmentation des surfaces boisées (fig. 2) par vieillissement des peuplements en place grâce au déroulement des successions végétales. Sur le secteur amont (fig. 2A), le taux de recouvrement de la végétation arborée passe de $17 \%$ en 1946 à $26 \%$ en 2000, la plus forte augmentation étant enregistrée jusqu'à la décennie 1970 (+35\% environ). Durant la période 1971-1985, de fortes pertes sont observées alors que la surface moyenne par taches reste la plus élevée de ces cinquante dernières années. Ensuite, à partir des années 1990, un nouvel accroissement du recouvrement forestier est enregistré, conjointement à la stabilisation spatiale des taches.

Sur le secteur intermédiaire (fig. 2B), les gains de surfaces forestières ont été progressifs. Les surfaces occupées par les boisements ont augmenté de $10 \%$ pour la même échelle de temps; la progression s'étant accélérée depuis les années 1970. Pratiquement absente en 1946, la végétation arborée occupe actuellement près de 120 ha et la position des taches se stabilise.

Sur le secteur aval (fig. 2C), la végétation arborée est la deuxième unité paysagère la plus représentée après les surfaces agricoles. Malgré une légère baisse du recouvrement en 1985 (proportion de 9,85\%), les surfaces occupées par les forêts ont augmenté de l'ordre de $31 \%$ entre 1949 et 2000 (respectivement $11,42 \%$ et $16,55 \%$ ). Alors que ses proportions de recouvrement ne varient pratiquement pas entre 1949 et 1971, la végétation arborée est par contre spatialement instable. Cette instabilité s'explique par les nombreuses coupes et éclaircissements de bois. À partir de 1985, l'instabilité spatiale décroît et les taches de végétation arborée conservent leur position.

\section{DYNAMIQUE DE L'OCCUPATION DE L'ESPACE PAR L'AGRICULTURE}

Avec une proportion fluctuant autour de $50 \%$ de la superficie totale, l'agriculture du secteur amont (fig. 2A), constituée de cultures intensives et de prairies grasses, est l'unité paysagère la plus représentée. Elle constitue l'élément structurant du paysage alluvial. Durant la période 1946-1954, sa plus forte progression est observée avec un gain de 5,03\%. Ensuite, à partir de la période 1971-1985, cette unité paysagère se stabilise spatialement et ses dimensions restent pratiquement identiques. À partir de 1993, ses proportions diminuent et son recouvrement atteint $43,05 \%$ en 2000.

Avec $39 \%$ du recouvrement en 1946 et $44 \%$ en 2000 , la matrice paysagère du secteur intermédiaire (fig. 2B) est également structurée par les cultures et les prairies grasses. Les surfaces de cultures ont fortement augmenté après 1954 conjointement à une stabilisation progressive de leur position. Pourtant, depuis le début des années 1990, la progression des cultures sur le milieu alluvial est stoppée et tend même à 
A) Secteur de Joze-Maringues

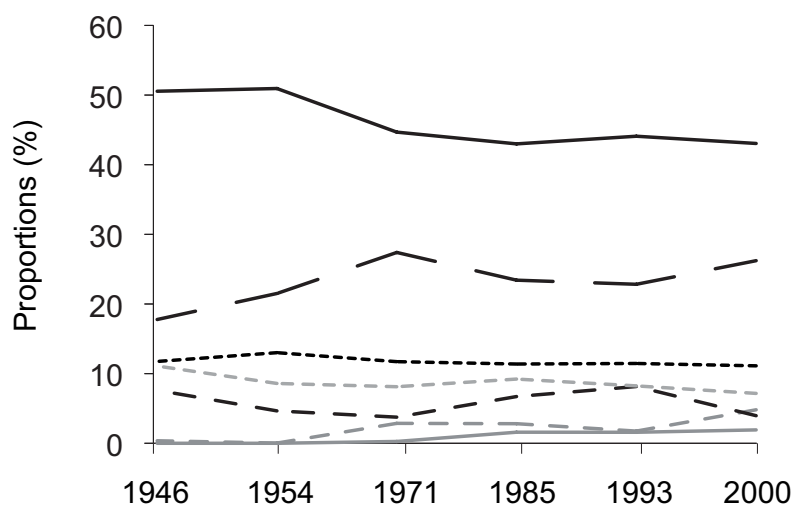

C) Secteur de Villeneuve-sur-Allier

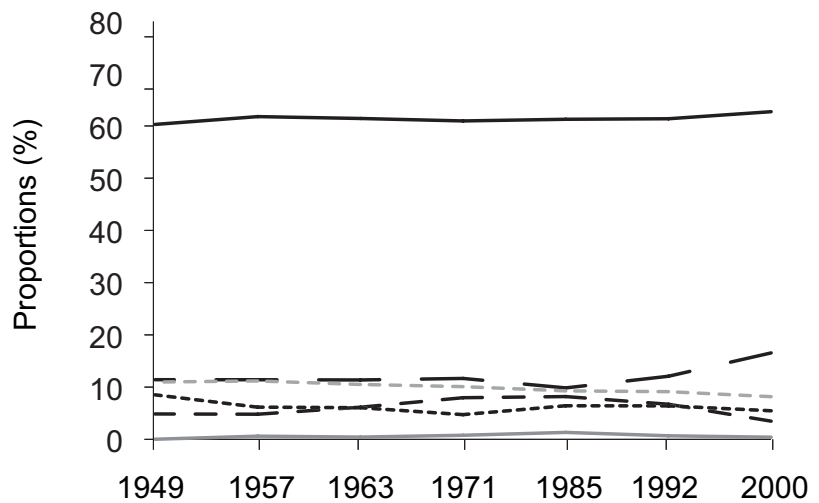

FIGURE 2. Évolution temporelle relative des différents types d'occupation du sol dans les secteurs de (A) Joze-Maringues, (B) Châtelde-Neuvre et (C) Villeneuve-sur-Allier.

régresser $(46,45 \%$ en $1992,45,80 \%$ en $1997,44,19 \%$ en 2000).

Sur le secteur aval (fig. 2C), à l'inverse des deux précédents secteurs, la matrice paysagère est une matrice dominée par le bocage dont les proportions fluctuent autour de $60 \%$ de la superficie totale. Après une progression de $1,5 \%$ entre 1946 et 1957, cette unité a quantitativement peu varié en surface. Seulement, cette faible extension correspond à une modification de l'utilisation de l'espace, c'est-à-dire à la transformation du bocage en champs de cultures intensives irriguées.

\section{APPARITION DE NOUVELLES UNITÉS PAYSAGÈRES}

Parallèlement au développement des cultures intensives et à l'extension des surfaces forestières, deux nouvelles unités paysagères apparaissent au début des années 1970: les plantations de peupliers et les extractions de granulats.

Le secteur amont est le plus concerné par l'émergence de ces nouvelles classes d'occupation du sol. La culture de peupliers hybrides est une activité qui n'a pas cessé de s'accroître

\section{B) Secteur de Châtel-de-Neuvre}

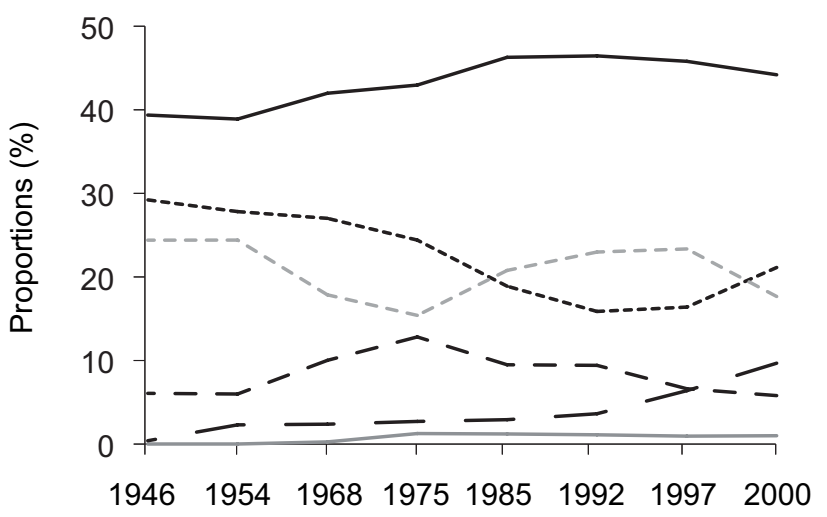

\section{Légende :}

$\begin{array}{ll}----- & \text { Bande active } \\ -\cdots-- & \text { Végétation herbacée } \\ --- & \text { Végétation arbustive } \\ - & \text { Végétation arborée } \\ - & \text { Cultures } \\ - & \text { Plantations } \\ -\ldots-- & \text { Gravières }\end{array}$

Relative temporal evolution of land use type in the (A) JozeMaringues, (B) Châtel-de-Neuvre and (C) Villeneuve-sur-Allier reaches.

sur le lit majeur aux dépens des surfaces herbacées, arbustives et arborées, conduisant ainsi à une banalisation du milieu. Ces plantations sont, pour la plupart, exploitées par coupes blanches laissant s'installer la friche pour être à nouveau plantées, bien que d'autres semblent avoir été abandonnées depuis plus de 40 ans (Chevallier, 1996). Cette activité n'atteint pas le même degré de développement dans les deux autres secteurs. Pour les secteurs aval et intermédiaire, les plantations datent des années 1950 et 1960 . Sur le secteur intermédiaire, les surfaces ont cessé d'augmenter en 1975. Certaines, insuffisamment entretenues, ont été colonisées par d'autres espèces et ont perdu leur physionomie spécifique.

Les extractions de granulats se sont essentiellement développées sur le secteur amont. Une première gravière est implantée dès 1967 dans le chenal principal au droit du méandre de Tissonnières. Deux autres concessions seront également autorisées dans le lit mineur. Sur le secteur intermédiaire, quelques extractions de granulats ont eu lieu au droit du méandre de Bessay-sur-Allier; elles correspondent sans doute à des emprunts effectués dans le passé par les services publics qui n'ont pas obligatoirement fait l'objet de déclaration (Centre d'Études Techniques de l'Équipement de 
Lyon, 1982). Aucune extraction n'a été conduite sur le secteur aval.

\section{DYNAMIQUE DE L'ACTIVITÉ ÉROSIVE}

Les résultats indiquent un maintien du dynamisme du chenal (fig. 3) au cours des cinquante dernières années pour l'ensemble des secteurs. Cependant, à l'échelle des intervalles de temps des photographies aériennes, l'activité érosive est contrastée. Pour le secteur amont (fig. 3A), une reprise de l'érosion est observée durant la période 1971-1985 tandis que, pour le secteur intermédiaire (fig. 3B), elle est effective lors des périodes 1975-1985 et 1992-1997. Cette recrudescence de l'activité érosive se traduit par une augmentation des valeurs centrales et de dispersion dont la forte variabilité traduit une érosion plus localisée.

\section{A) Secteur de Joze-Maringues}

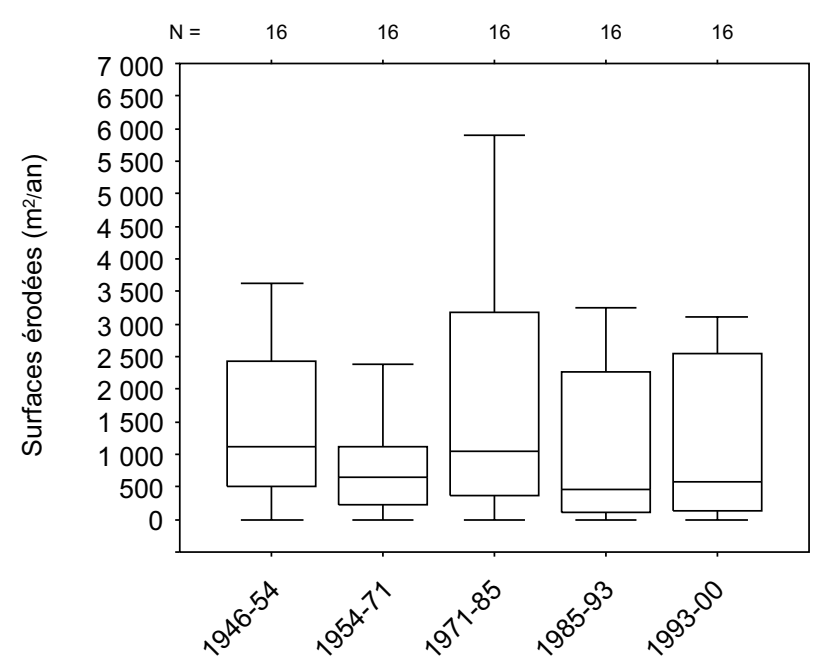

C) Secteur de Villeneuve-sur-Allier

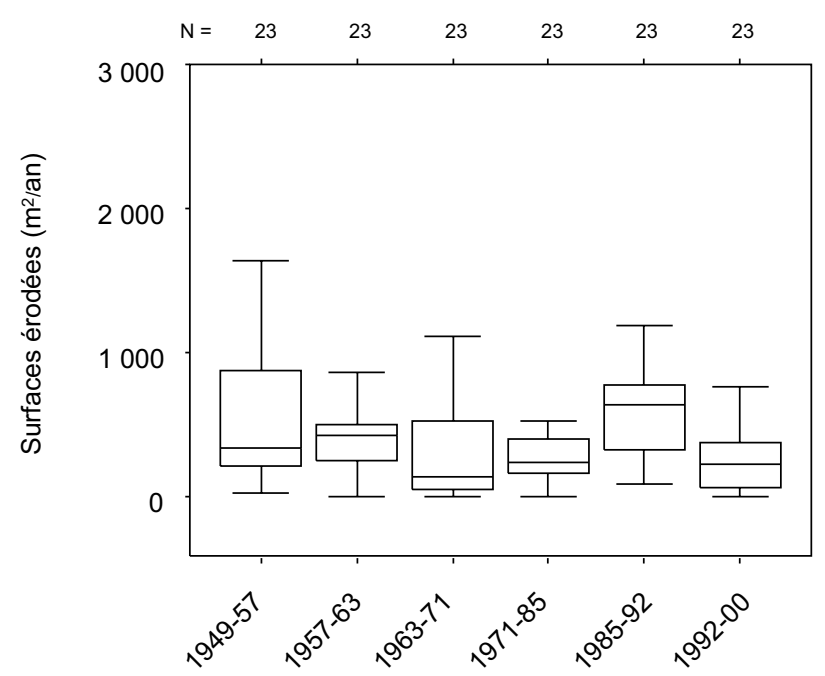

FIGURE 3. Évolution des surfaces érodées entre 1946 et 2000 (en $\mathrm{m}^{2} / \mathrm{an}$ ) sur les secteurs de (A) Joze-Maringues, (B) Châtel-de-Neuvre et (C) Villeneuve-sur-Allier.
Les taux d'érosion sont également différents d'un secteur à l'autre. Sur le secteur amont, à l'échelle des cinquante dernières années, l'Allier a érodé à un rythme de $0,18 \mathrm{ha} / \mathrm{km} / \mathrm{an}$. Sur le secteur intermédiaire, avec 0,58 ha/km/an, le taux d'érosion estimé entre 1946 et 2000 est proche de celui mesuré pour l'ensemble de la Réserve Naturelle du Val d'Allier par Luherne (1994) (0,6 ha/km/an entre 1946 et 1992). Enfin, avec $0,10 \mathrm{ha} / \mathrm{km} / \mathrm{an}$, le secteur aval est celui dont les taux d'érosion sont les plus faibles. Dans le même temps, les processus érosifs affectent de manière inégale les unités paysagères. Sur le secteur amont, $35 \%$ (38 ha) de la végétation arborée et $30 \%$ (33 ha) de la végétation herbacée ont été érodés. La forte proportion de boisements érodée s'explique à la fois par son fort taux de recouvrement (elle occupe près du quart de la surface totale d'étude) et par sa localisation aux abords de la bande active. Quant à la végétation arbustive et aux cultures,

\section{B) Secteur de Châtel-de-Neuvre}

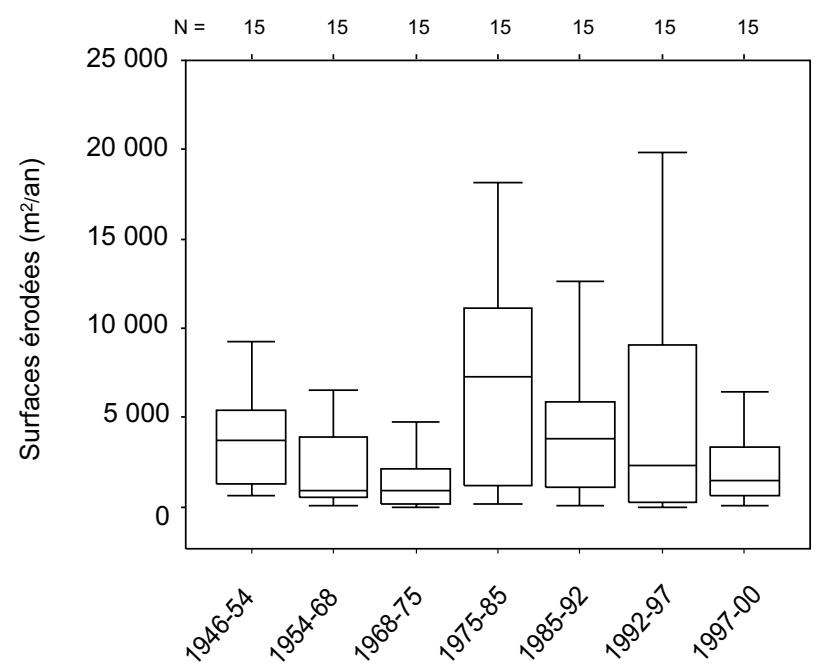

Légende :

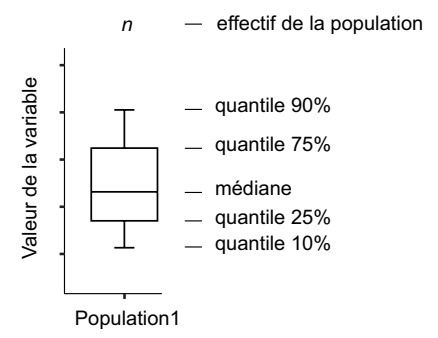

Evolution of eroded surfaces between 1946 and 2000 (in $\mathrm{m}^{2} /$ year) in the (A) Joze-Maringues, (B) Châtel-de-Neuvre and (C) Villeneuvesur-Allier reaches. 
leurs proportions érodées sont deux fois moins fortes avec respectivement $15,12 \%$ (16 ha) et 18,12\% (19 ha) ; les surfaces de cultures érodées subissant une forte décroissance au fil du temps.

Sur le secteur intermédiaire et contrairement au secteur amont, plus de $63 \%$ (207 ha) des zones d'érosion ont concerné la végétation herbacée, soit environ quatre fois plus que la végétation arbustive (16,36 \%, 53,77 ha) et les cultures (18\%, 59 ha). Avec une augmentation des surfaces érodées de près de $7 \%$ depuis 1946, la proportion de végétation arborée érodée reste faible mais, parallèlement à son extension spatiale, elle subit davantage les processus érosifs, signe d'une localisation de plus en plus proche du chenal actif. La végétation arbustive suit une évolution identique et, corrélativement à son extension, ses proportions érodées augmentent, passant de 8,46\% en 1946 à 24,24\% en 1985, date de son maximum de développement.

Sur le secteur aval, avec $41 \%$ de sa superficie totale (24 ha), la végétation arborée est l'unité paysagère la plus érodée à cause de sa forte implantation de part et d'autre du chenal actif et sur les îles. Puis, par ordre décroissant de contribution, les autres unités érodées sont la végétation herbacée avec $39 \%$ de sa superficie totale (23 ha) et la végétation arbustive dont la proportion avoisine les $17 \%$ (environ 10 ha). Les autres unités ne sont pas touchées par les variations des taux d'érosion.

\section{DYNAMIQUE DE LA BANDE ACTIVE}

Les résultats présentés témoignent d'une réduction générale de la bande active depuis la seconde moitié du $20^{\mathrm{e}}$ siècle au profit des unités du lit majeur (fig. 4). Cette tendance se manifeste pourtant selon des intensités et des rythmes différents sur les trois secteurs.

Sur le secteur amont, la bande active est passée de 122 ha en 1946 à 79 ha en 2000, soit respectivement de $11 \%$ à $8,30 \%$ de la surface d'étude. Quant à la largeur moyenne, elle a diminué de l'ordre de $56 \%$ (de 102,05 m en 1946 à $65,36 \mathrm{~m}$ en 2000 ) au profit de la végétation pionnière. Le test a posteriori de Scheffé montre des largeurs de bande active significativement différentes $(p<0,05)$ entre les années 1946 , 1985 et 2000 alors que les périodes 1954-1971, 1971-1992 et 1992-2000 ne dévoilent pas de différences significatives $(p>0,05)$. Ces résultats mettent en évidence trois tendances (fig. 4A). D'abord, une diminution de la largeur de la bande active entre 1946 et 1954 au profit de la végétation herbacée et arbustive. Puis, la largeur de la bande active semble se stabiliser: une phase de plateau est enregistrée jusqu'au début des années 1990 durant laquelle la tendance centrale est stable. L'année 1985 correspond cependant à une augmentation des distributions statistiques des largeurs de bande active. Cet accroissement est attribué à une hausse de la largeur moyenne et à une augmentation des fortes valeurs aux dépens de la végétation herbacée et arborée. Enfin, à partir de 1992, la largeur de la bande active diminue à nouveau et passe de 100 à 79 ha $(9,22 \%$ à $8,25 \%)$. Cette tendance semble correspondre à un réajustement de la largeur de la bande active avec un retour à la tendance observée antérieurement à 1985. Aujourd'hui, l'étendue des largeurs est nettement plus faible qu'en 1946, ceci s'explique par la disparition des fortes valeurs et la diminution de la valeur médiane.

Sur le secteur intermédiaire, la bande active a diminué faiblement. Elle est passée de 295 ha en 1946 à 213 ha en 2000, soit respectivement $24 \%$ et $17 \%$ de la surface d'étude. Les résultats de l'analyse indiquent une baisse de la largeur moyenne de l'ordre de $37 \%$ (de 249,92 m en 1946 à $182,09 \mathrm{~m}$ en 2000) et son évolution durant les cinquante dernières années est contrastée (fig. 4B). Le test a posteriori de Scheffé indique un fonctionnement identique entre les années 1946 et 1954, puis montre que les changements se produisent essentiellement entre 1968 et 1975 ( $p<0,05)$ alors que les périodes 1946-1954 et 1985-1997 ne dévoilent pas de différence significative $(p>0,05)$. Deux tendances sont observables. Une première de 1946 à 1975, où la bande active se contracte; elle passe de 295 à 186 ha, soit une diminution surfacique de $58 \%$ et de $72 \%$ de la largeur moyenne au profit presque exclusivement de la végétation herbacée qui, dans le même temps, se transforme par déroulement classique des successions végétales. Puis, une seconde tendance est enregistrée. Elle est marquée par l'augmentation de la largeur de la bande active jusqu'en 1992 (+38\% pour la largeur moyenne). Entre 1975 et 1997, la bande active passe de 186 à 282 ha aux dépens de la végétation herbacée et arbustive. À partir de 1997, une nouvelle phase de contraction débute et se poursuit jusqu'en 2000. Cette évolution contrastée se traduit également par une forte variabilité de la dispersion des valeurs de la bande active: là où les valeurs centrales sont les plus fortes (1946-1954, 1985-1992 et 1992-1997), la dispersion est la plus forte. Cette caractéristique renseigne sur la présence de quelques tronçons à large bande active.

Sur le secteur aval, la bande active a enregistré une diminution de $27 \%$ de la largeur moyenne entre 1946 et 2000 (de $194,83 \mathrm{~m}$ à $141,81 \mathrm{~m}$ ) et sa surface est passée de 230 à 172 ha, soit respectivement $11 \%$ et $8 \%$ de la surface d'étude. Les valeurs centrales et de dispersion de la largeur de bande active ont enregistré une baisse continue et progressive (fig. 4C). Néanmoins, la rétraction semble avoir véritablement débuté durant les années 1970. Le test a posteriori de Scheffé montre en effet un comportement identique entre les années 1949, 1957 et 1963 ( $p>0,05$ ) et indique des changements significatifs $(p<0,05)$ entre ces mêmes années et la période 1985-1992. Sur ce dernier secteur, la bande active est d'abord remplacée par la végétation pionnière herbacée et arbustive puis, à partir des années 1990, par la végétation forestière. Par ailleurs, cette évolution se traduit par une diminution de l'étendue des largeurs de bande active et les valeurs de dispersion sont beaucoup plus faibles en 2000 qu'en 1949.

En valeur relative (tabl. V), la réduction de la surface de la bande active a été de $56 \%$ sur le secteur amont, de $37 \%$ sur le secteur intermédiaire et de $27 \%$ sur le secteur aval. Sur ce dernier secteur, 61,6 ha ont été colonisés par la végétation, ce qui représente $0,10 \mathrm{ha} / \mathrm{km} / \mathrm{an}$, contrairement à $0,068 \mathrm{ha} / \mathrm{km} / \mathrm{an}$ sur le secteur amont et $0,11 \mathrm{ha} / \mathrm{km} / \mathrm{an}$ sur le secteur intermédiaire. À l'échelle des trois sites étudiés, ceci 


\section{A) Secteur de Joze-Maringues}

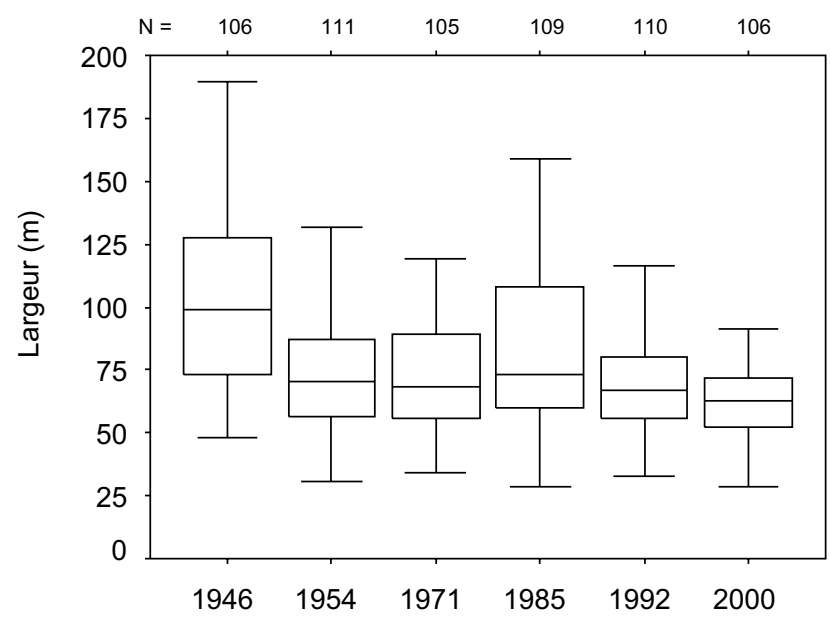

C) Secteur de Villeneuve-sur-Allier

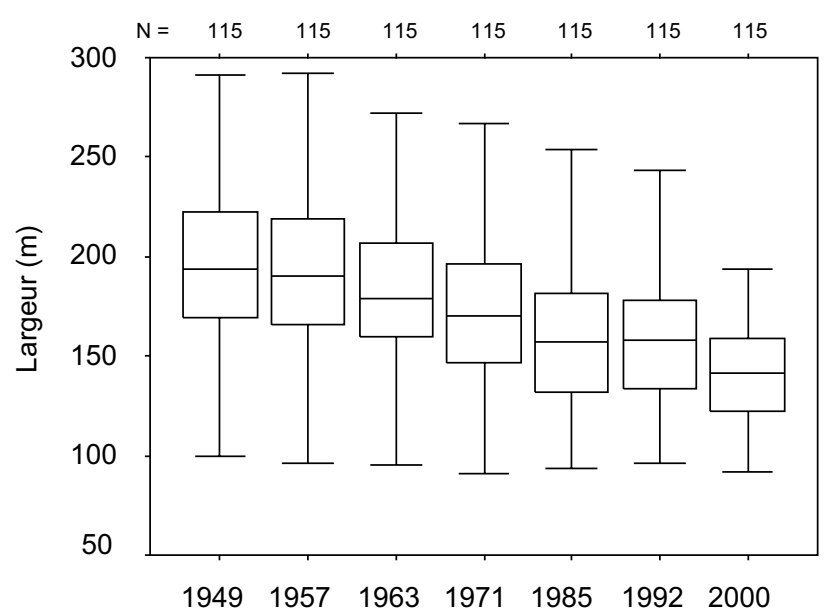

FIGURE 4. Évolution de la largeur de la bande active entre 1946 et 2000 (en m) des secteurs de (A) Joze-Maringues, (B) Châtel-deNeuvre et $(C)$ Villeneuve-sur-Allier.

signifie que le tiers jusqu'à la moitié des surfaces d'activité de la rivière s'est stabilisé durant la période étudiée.

\section{DISCUSSION}

En s'appuyant sur le modèle de fonctionnement géomorphologique des bassins versants issu de la littérature (Piégay et Schumm, 2003) et sur les facteurs expliquant la rétraction des bandes actives du bassin rhodanien (Liébault et Piégay, 2002) (tabl. I), la présente analyse, fondée sur une comparaison de trois sites, permet de tester successivement, grâce à une approche déductive, les causes potentielles responsables des modifications de la plaine d'inondation.

La théorie de la hiérarchie (Allen et Starr, 1982) conduit à replacer les observations hydrologiques dans un cadre plus vaste que celui de la seconde moitié du $20^{\mathrm{e}}$ siècle. Depuis le

\section{B) Secteur de Châtel-de-Neuvre}

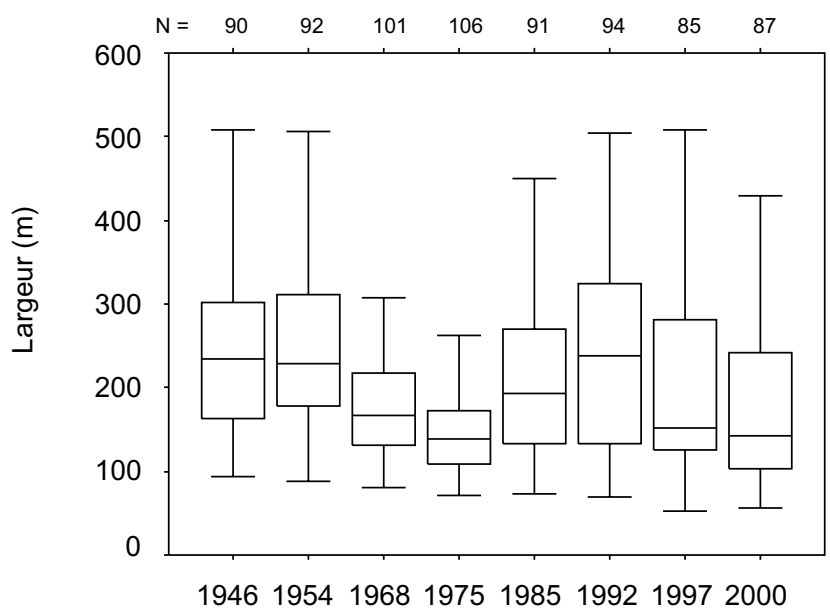

\section{Légende :}

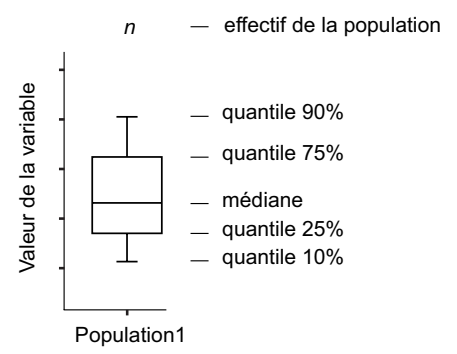

Evolution of active channel width between 1946 and 2000 (in $\mathrm{m}$ ) in the (A) Joze-Maringues, (B) Châtel-de-Neuvre and (C) Villeneuve-surAllier reaches.

milieu du $19^{e}$ siècle, Gautier et al. (2000) évoquent une réduction de la fréquence et de l'intensité des crues de l'Allier. Les modifications des conditions hydrologiques et hydrauliques provoquent une diminution des contraintes liées à l'excès d'eau et d'énergie et se traduisent par une augmentation du recouvrement ligneux dans la bande active (Piégay et al., 2003a). Cette réduction historique de l'hydraulicité participe vraisemblablement au phénomène de recolonisation végétale, mais ne semble pas être à l'origine de la végétalisation récemment observée. Dans le cas d'une réduction de l'hydraulicité, le chenal aurait réagi instantanément à la baisse des débits (Peiry et Vivian, 1994), et non entre 1946 et 2000. La rétraction de la bande active se serait alors manifestée au début du $20^{\mathrm{e}}$ siècle; or, les cartes d'état-major montrent encore un chenal tressé en 1904. Si des études ont auparavant montré (Bravard, 1991; Gautier, 1992) que la végétali- 


\section{TABLEAU V}

Changements ayant affecté les surfaces de la bande active entre 1946 et 2000 sur les secteurs de Joze-Maringues, Châtel-de-Neuvre et Villeneuve-sur-Allier

\begin{tabular}{|c|c|c|c|c|c|c|}
\hline \multirow[t]{2}{*}{ Secteur } & \multicolumn{3}{|c|}{$\begin{array}{l}\text { Superficie de la bande active } \\
\text { (ha) }\end{array}$} & \multicolumn{2}{|c|}{$\begin{array}{l}\text { Longueur axiale de la bande active } \\
\qquad(\mathrm{km})\end{array}$} & \multirow{2}{*}{$\begin{array}{c}\begin{array}{c}\text { Taux d'érosion } \\
\text { annuel } \\
\text { (ha/km/an) }\end{array} \\
1946-2000\end{array}$} \\
\hline & 1946 & 2000 & $\begin{array}{c}\text { Diminution } \\
(\%)\end{array}$ & 1946 & 2000 & \\
\hline Joze-Maringues & 109,9 & 70,79 & 56 & 10,65 & 10,65 & 0,068 \\
\hline Châtel-de-Neuvre & 229,7 & 170,1 & 37 & 9,215 & 9,109 & 0,11 \\
\hline Villeneuve-sur-Allier & 224,0 & 162,4 & 27 & 11,47 & 11,44 & 0,10 \\
\hline
\end{tabular}

sation des têtes de bassins à la fin du $19^{\mathrm{e}}$ siècle pouvait expliquer les changements morphologiques du chenal dans la mesure où elle contribuait à retenir la charge sédimentaire sur les versants, cette dynamique qui se manifeste loin des présents sites d'étude, ne peut pas être retenue pour expliquer les phénomènes observés sur ces sites. Comment peut-elle expliquer la maturation progressive de la végétation de la plaine d'inondation et la rétraction de la bande active au cours des cinquante dernières années compte tenu du temps nécessaire à la charge pour transiter jusqu'à ces secteurs de l'Allier? Les contrôles locaux semblent donc prépondérants pour expliquer les changements paysagers du corridor fluvial.

\section{MODIFICATION DES PRATIQUES TRADITIONNELLES}

De toute évidence, l'évolution de l'occupation du sol de la plaine d'inondation de l'Allier est forcément indépendante des processus affectant le chenal. Le contrôle anthropique apparaît être un facteur clé pour expliquer l'extension progressive de la végétation dans la zone de contact entre le lit mineur et le lit majeur.

Sur les bords de l'Allier, jusqu'à la Seconde Guerre mondiale, les pratiques agro-pastorales ont permis le maintien d'un paysage ouvert composé de pâturages et de broussailles. Sur les secteurs amont et intermédiaire, l'agriculture se révélait correspondre à un pâturage relativement intensif sur les grèves, les îles et les parties enherbées exemptes de cultures. Le paysage près du chenal était dominé par des zones graveleuses ou enherbées. Sur le secteur aval, la mosaïque paysagère correspondait au bocage bourbonnais à mailles larges, complanté d'arbres, de taillis et de forêts avec habitat dispersé. Le paysage des marges de la bande active était encore composé de graviers et de prairies avec une végétation riveraine arbustive et arborée déjà notablement établie.

Dans les années 1960, la France a bâti une politique agricole pour moderniser les exploitations et les techniques, et ouvrir cette économie vers l'extérieur afin de produire massivement des denrées agricoles pour nourrir une population en croissance (Michelin, 1995). Ce développement s'est entre autre appuyé sur l'intensification des productions agricoles et la transformation du territoire. Les modèles de production agricole intensive développés depuis les années 1960 ont ainsi transformé la terre en un espace de plus en plus uniforme et aménagé pour être l'objet de pratiques culturales normées (Alphandéry, 2004). Cette intensification de l'agriculture, qui se traduit par une augmentation des surfaces agricoles et un changement des pratiques culturales sur le lit majeur, est une tendance générale observée dans les trois secteurs. La transformation de l'occupation du sol est confirmée par l'augmentation des surfaces irriguées sur les communes appartenant aux trois secteurs d'étude; l'irrigation s'étant affirmée comme le moyen de conduire une agriculture céréalière productiviste et concurrentielle (Lelli, 2004).

Malgré tout, la transformation de l'espace ne s'est pas produite simultanément et selon la même intensité sur les trois secteurs. Le secteur intermédiaire est le premier à avoir connu une transformation des pratiques culturales. Dans le Val d'Allier, du fait de la qualité des terres agricoles et l'étendue de sa plaine d'inondation, cette modification a débuté dès 1955 avec les premiers essais de culture de maïs (Lemaire, 1996). Ce n'est que depuis les années 1970 et surtout en rive droite que les grandes cultures irriguées (maïs, tournesol, colza, pois fourragers) se sont véritablement développées; restructurant le parcellaire aux dépens des zones en herbe, des haies et d'anciens bras petit à petit bouchés. Ces pratiques expliquent l'agrandissement des parcelles et ont participé à la géométrisation du paysage agricole (Lelli, 2004). Parallèlement, la pression humaine sur le milieu s'est traduite par une augmentation des voies d'accès et la réduction du réseau de haies. Pour le secteur amont, le développement de l'agriculture céréalière a été moins intense, mais semble intervenir dès les années 1950, puis dans les années 1970. En 2000, dans ces deux secteurs, la proportion de cultures est sensiblement la même, mais toutes deux ont enregistré une légère diminution à partir des années 1990 au profit de la végétation herbacée et arborée, dans le secteur amont vraisemblablement par progression des lisières. Cette légère baisse peut sans doute s'expliquer par des changements socio-économiques. Quant au secteur aval, sa physionomie a très peu changé en cinquante ans et le bocage bourbonnais prédomine. Seules quelques surfaces de grandes cultures irriguées se sont développées dans la partie amont en rive droite depuis les années 1980.

Les changements des pratiques culturales se sont donc traduits par une intensification agricole par conversion à la culture intensive sur le lit majeur et une régression du pasto- 
ralisme extensif et des coupes de bois aux abords du lit mineur. La réduction de l'intensité des pratiques traditionnelles, voire leur abandon, a eu pour conséquence de favoriser l'extension progressive du recouvrement arbustif, puis arboré dans la zone de contact entre le lit mineur et le lit majeur même dans les secteurs à fort dynamisme latéral du chenal (secteur intermédiaire). Sur le secteur aval, malgré quelques modifications structurelles et d'utilisation du sol, le bocage bourbonnais reste dominant. Toutefois, à cause de la diminution de la pression humaine sur les boisements, les marges de la bande active se boisent par extension des taches arborées existantes.

Le secteur intermédiaire se distingue des sites amont et aval par le maintien d'une activité pastorale. Même si l'intensité du pâturage est plus faible que dans les années 1940, celui-ci perdure notamment aux abords de la bande active. La présence des prairies expliquerait la dynamique géomorphologique contemporaine. Le maintien d'une activité pastorale limiterait donc le développement de la végétation riveraine et éviterait la rétraction de la bande active; cette stabilité étant entretenue par le contexte morphologique. Cette hypothèse se justifie au regard de l'évolution de la basse vallée du Doubs (Liébault et Piégay, Piégay, comm. pers.) dont les marges ont continué à être valorisées à des fins pastorales et où la bande active ne s'est pas rétractée. Cependant, malgré le maintien d'un pâturage extensif, la pression sur la végétation s'est relâchée et a pu initier le processus de végétalisation, puis l'augmentation des surfaces boisées. Néanmoins, le recouvrement forestier reste faible en lit majeur et les changements d'occupation du sol ne sont pas encore suffisamment spectaculaires pour qu'ils exercent un contrôle sur la morphologie du lit comme celui observé sur les rivières du sud-ouest de la France.

Comme sur l'Ain (Dufour, 2005), la déprise agro-pastorale, apparaît donc être le facteur dominant pour expliquer la progression des boisements sur la plaine d'inondation.

\section{RÉDUCTION DES APPORTS SÉDIMENTAIRES}

S'il existe un déficit sédimentaire lié à des facteurs agissant à l'amont, celui-ci devrait se propager de l'amont vers l'aval. Les réponses morphologiques des sites, notamment la rétraction du lit par végétalisation, devraient alors être décalées dans le temps. Ce n'est pas le cas sur la plaine alluviale de l'Allier, car le secteur amont ne se végétalise pas plus précocement que le secteur aval. Comme en témoignent les cartes d'état-major de 1904, le changement de style fluvial semble avoir eu lieu entre le début du $20^{e}$ siècle et 1946 (date du premier cliché aérien disponible), hormis pour le secteur intermédiaire pour lequel le tressage est encore présent en 1946. Malheureusement, aucune donnée n'est disponible entre 1904 et 1946 pour vérifier cette hypothèse.

Dans le cas d'une réduction des apports sédimentaires locaux associés notamment à des extractions, l'extension des boisements en lit mineur a lieu de préférence dans les secteurs incisés. À l'échelle de l'Allier inférieur (fig. 1), l'étude de la DIREN Auvergne-Epteau (1998), qui synthétise l'ensemble des données disponibles, atteste qu'environ $70 \%$ de l'Allier aurait subi une incision de l'ordre de 0,5 à 3,5 m, avec une valeur médiane se situant autour de 1,2 $\mathrm{m}$. Au sein du réseau hydrographique, trois zones d'activité extractive majeures ont été identifiées. Ces zones se situent à l'amont de chacun des trois sites d'étude. Par leur situation, elles peuvent sans doute contribuer localement à un déficit sédimentaire. La réduction de la bande active par végétalisation pourrait alors s'expliquer par ce déficit. Ce facteur a été particulièrement bien étudié dans les rivières de piémont. Sur l'Isère, en amont de Grenoble, les extractions de granulats, en favorisant la diminution du transport grossier, sont à l'origine de l'enfoncement du chenal, de la fixation des formes fluviales et de leur végétalisation (Vautier, 2000). Selon l'étude de la DIREN AuvergneEpteau (1998), le secteur amont semble s'inciser tandis que nos observations de terrain attestent un enfoncement du chenal sur le secteur aval. II semble donc que le déficit des apports sédimentaires locaux soit un facteur à l'origine de la contraction de la bande active sur les secteurs amont et aval. Le secteur intermédiaire, sur lequel la diminution de la largeur de la bande active est la moins nette, semble globalement en phase d'exhaussement (DIREN Auvergne-Epteau 1998). La correspondance incision-rétraction sur les secteurs amont et aval et exhaussement-stabilité latérale sur le secteur intermédiaire donne une certaine validité au fait que l'incision locale puisse être considérée comme un facteur responsable du processus de végétalisation du lit.

Alors que le déficit sédimentaire a un impact sur la largeur de la bande active, il a en revanche un effet moindre sur les rythmes érosifs qui ont peu évolué au cours des cinquante dernières années.

\section{RÉDUCTION DE L'HYDRAULICITÉ}

Une diminution de l'hydraulicité peut être à l'origine d'une rétraction de la bande active bien que sur l'Ardèche, le Buech ou encore l'Ouvèze, aucune baisse du régime de crue n'est effectivement observée alors que le lit mineur enregistre une rétraction (Dufour et Piégay, 2006). Si l'accroissement végétal est lié à la réduction de l'hydraulicité, cette extension devrait être synchrone sur les trois secteurs d'étude et correspondre à une période de faible hydraulicité. Or, l'analyse de la chronologie des débits journaliers maximaux (fig. 5) ne montre pas de diminution de l'hydraulicité. En fait, l'augmentation des surfaces boisées se manifeste réellement sur les trois secteurs à partir des années 1980 (fig. 2), même si le recouvrement ligneux diminue sur le secteur amont durant les années 1970 en raison de la mise en place de plantations de peupliers et l'ouverture de sites d'extraction. Cette colonisation est synchrone d'une période de plus forte hydraulicité (augmentation des crues dont le débit est supérieur au $Q_{2}$ durant la décennie 1980 et le milieu des années 1990 (fig. 5)). À l'échelle de temps considérée, le régime hydrologique ne semble donc pas être un facteur déterminant pour expliquer l'extension de la végétation arbustive et des boisements sur la plaine d'inondation. Malgré tout, dans les sites nouvellement créés des secteurs amont et intermédiaire, dont la dynamique est indépendante des activités humaines, les fluctuations 
A) Débit journalier maximum $\left(\mathrm{m}^{3} / \mathrm{s}\right)$

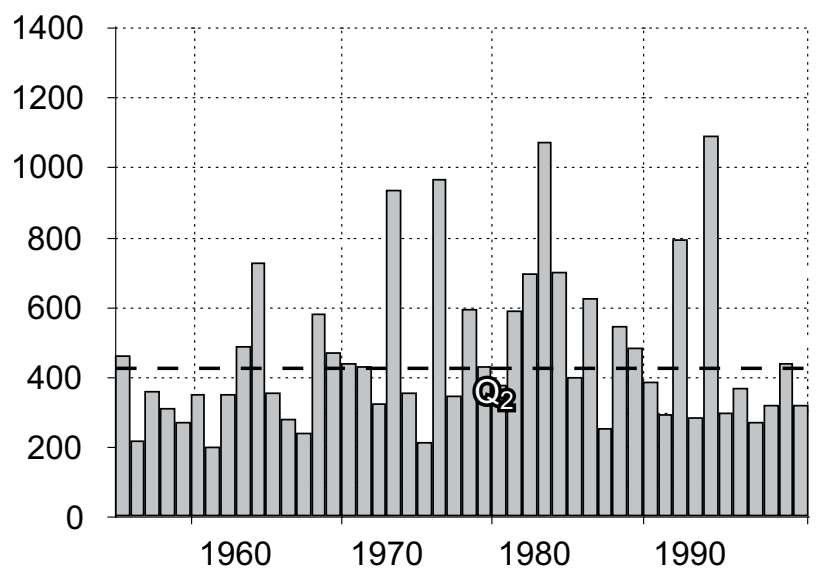

FIGURE 5. Débits journaliers maximum aux stations (A) Vic-le-Comte (1955-2000) et (B) Moulins (1969-2000) (Source: banque HYDRO).
B) Débit journalier maximum $\left(\mathrm{m}^{3} / \mathrm{s}\right)$

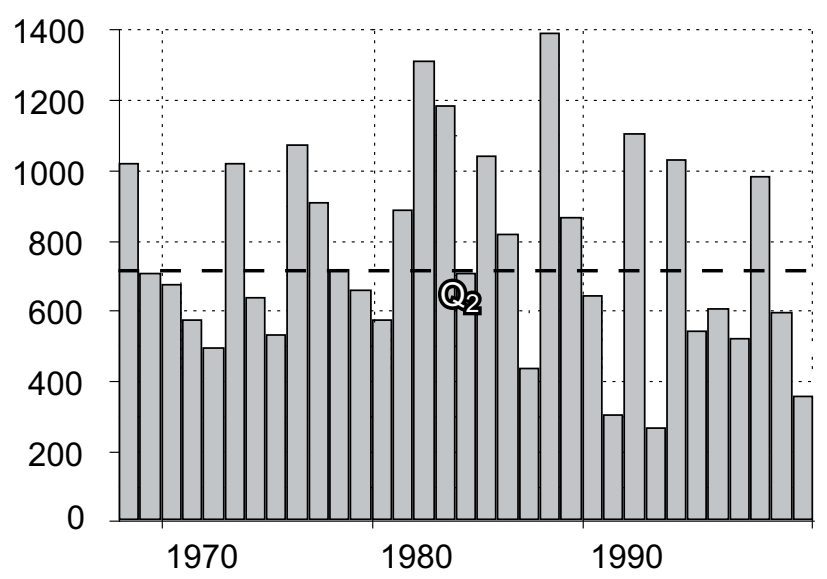

Maximum daily discharges at (A) Vic-le-Comte (1955-2000) and (B) Moulins (1969-2000) water stations (Source: HYDRO database). internes des débits maximaux annuels peuvent sans doute initier localement la progression de la végétation pionnière, herbacée et arbustive.

En revanche, le rythme de crues semblent régir l'érosion latérale et la largeur de la bande active, en particulier sur le site amont et de façon plus singulière sur le site intermédiaire (fig. 3A, B et 4A, B). Le contrôle géomorphologique est fort dans le secteur intermédiaire et s'exprime essentiellement en amont du méandre de Bessay-sur-Allier, où paradoxalement la végétation arbustive et les boisements ont le plus progressé à partir des années 1970 (fig. 2B). Ainsi, l'augmentation de l'hydraulicité s'est traduite par un nouvel élargissement de l'ensemble de la partie amont et par une légère modification de la structure du corridor végétal aux abords de la bande active. Mais, c'est sans doute la juxtaposition de ce régime de crue et d'un contexte géomorphologique particulier avec une plus forte dynamique de méandrage durant les décennies 1980 et 1990 qui explique cette instabilité. En fait, l'augmentation de l'hydraulicité s'est manifestée simultanément à deux recoupements successifs du méandre de Bessay-surAllier. Le premier recoupement date de 1980 et correspond à un re-calibrage du méandre effectué par les services de l'État. Le second est un recoupement naturel et total provoqué par une crue décennale en 1994. Ces recoupements illustrent le concept d'ajustement dynamique et de réponse complexe (Schumm, 1977). Ils ont entraîné un nouveau dynamisme de la bande active contribuant à accroître les surfaces érodées. La variabilité des largeurs de la bande active et des taux d'érosion peut donc s'interpréter comme le rétablissement progressif d'une situation d'équilibre dynamique après une courte période de changement à la suite du recoupement artificiel du méandre en 1980; ce retour à un équilibre progressif étant retardé par le recoupement de 1994 (fig. 3B, 4B).

\section{CONCLUSION}

Les résultats de cette étude attestent une diminution progressive et générale de la dynamique latérale du chenal, même si la réduction est moins nette sur le secteur intermédiaire. Ils indiquent également que les formations boisées gagnent progressivement du terrain sur la plaine d'inondation de l'Allier. Cette expansion de la végétation ligneuse, déjà évoquée il y a une vingtaine d'années (Fédération de la Région Auvergne pour la Nature et l'Environnement, 1982) mais non quantifiée, reste néanmoins faible puisque, pour l'ensemble des trois secteurs étudiés, le pourcentage de végétation arborée est passé de 10 à $18 \%$ en cinquante ans. L'observation des cartes d'occupation du sol atteste un développement des surfaces boisées de plus en plus proche du chenal actif, surtout pour le secteur le plus dynamique (secteur intermédiaire). Cette extension est non négligeable puisqu'elle pourrait, entre autres, modifier les conditions hydrauliques sur la plaine d'inondation (Tal et al., 2004) et contribuer à la production de bois flotté dans le système fluvial (voir Piégay, 2000).

II semble que les changements des pratiques culturales traditionnelles (coupes de bois, changements d'occupation du sol) soient à l'origine de la végétalisation du paysage alluvial dont le mécanisme est apparemment stimulé par la réduction des apports sédimentaires locaux, dans le contexte général de réduction de l'intensité des processus géomorphologiques d'origine naturelle (fin du Petit Âge Glaciaire) ou anthropique (extraction de granulats, enrochements).

L'augmentation des surfaces boisées se rencontre dans deux contextes géographiques et s'explique par les particularités morphologiques et anthropiques locales qui contrôlent le développement de la végétation ligneuse et sa répartition sur la plaine d'inondation:

(1) Sur le secteur disposant encore d'un espace de divagation latérale (secteur intermédiaire), la végétation ligneuse se développe depuis la fin des années 1970. Les zones de prairies ont régressé depuis les cinquante dernières années au profit des boisements, mais elles sont encore largement présentes. La progression des ligneux s'effectue préférentiellement dans les zones récemment abandonnées par le che- 
nal, par les activités humaines traditionnelles et dans les zones inadaptées à la pratique d'une culture intensive irriguée;

(2) Sur les secteurs ne disposant que d'un espace de divagation limité à cause des enrochements (secteur amont) ou du style fluvial en anabranches (secteur aval), le phénomène de végétalisation est moins marqué et plus progressif. Sur le secteur amont, l'extension des ligneux se fait à partir de la forêt existante, mais semble avoir été favorisée par la diminution de la dynamique latérale du chenal (enrochement) et l'abandon progressif des activités de pâturage qui laisse des espaces ouverts colonisés par le bois. Dans le secteur en anabranches, le bocage traditionnel se maintient et la végétation ligneuse se développe dans le lit mineur d'une part, par extension des boisements déjà en place et, d'autre part, sur les îles davantage stables.

La diminution ou l'abandon des pratiques agro-pastorales traditionnelles apparaît donc être un facteur clé pour expliquer l'extension progressive de la végétation ligneuse dans la zone de contact entre le lit mineur et le lit majeur. Quant au comportement de la bande active, il semble s'expliquer à la fois par les activités de pâturage et les déficits sédimentaires locaux, ces derniers ayant un moindre effet sur les rythmes d'érosion. Sur le secteur intermédiaire dont le recouvrement prairial est encore important et dans un contexte d'une forte dynamique de méandrage, la largeur de la bande active reste stable. Par contre, sur le secteur aval, dans un contexte d'incision du chenal liée à la réduction des apports locaux et d'une quasi-disparition des zones de prairies, la bande active se rétracte.

Bien que ce travail repose sur une étude diachronique détaillée de l'évolution du paysage alluvial au cours des cinquante dernières années, un des problèmes fondamentaux est néanmoins celui de l'identification des perturbations et de l'évaluation de leurs effets (Pickett et al., 1989 cité dans Pautou et al., 1997). Parce que les systèmes fluviaux sont en perpétuelle évolution, faire le lien entre les changements morphologiques observés et les facteurs qui les contrôlent (activités anthropiques, hydrologie, dynamique sédimentaire, activités socio-économiques, etc.) reste compliqué. Dans ce contexte, l'analyse géographique comparée constitue un cadre méthodologique pertinent.

\section{REMERCIEMENTS}

L'auteur remercie Jean-Luc Peiry, Johannes Steiger et Franck Vautier pour leurs remarques ainsi que les lecteurs anonymes pour leurs suggestions et leurs corrections. Enfin, je remercie vivement Hervé Piégay pour l'intérêt qu'il a porté à ce travail et ses commentaires constructifs.

\section{RÉFÉRENCES}

Allen, T.H.F. et Starr, T.B., 1982. Hierarchy, perspectives for ecological complexity.

Alphandéry, P., 2004. L'agriculture française à l'heure de l'environnement et la gestion des territoires, p. 128-150. In Le défi du paysage, un projet pour l'agriculture. Les cahiers de la compagnie du paysage, Seyssel.
Amoros, C., Bravard, J.-P., Reygrobellet, J.-L., Pautou, G. et Roux, A.-L., 1988. Les concepts d'hydrosystème et de secteur fonctionnel dans l'analyse des systèmes fluviaux à l'échelle des écocomplexes. Bulletin d'écologie, 19: 531-546.

Amoros, C., Roux, A.L., Reygrobellet, J.L., Bravard, J.P. et Pautou, G., 1987. A method for applied ecological studies of fluvial hydrosystems. Regulated Rivers, 1: 17-36.

Bendix, J. et Hupp, C.R., 2000. Hydrological and geomorphological impacts on riparian plant communities. Hydrological processes, $14: 2977-2990$.

Bravard, J.-P., 1985. Le Haut-Rhône français, dynamique naturelle et impacts des travaux d'aménagement d'un fleuve et de sa vallée. Thèse de doctorat d'État, Université Jean Moulin Lyon III, 800 p.

Bravard, J.-P., 1991. La dynamique fluviale à l'épreuve des changements environnementaux: quels sont les enseignements applicables à l'aménagement des rivières? La Houille Blanche, 7-8: 515-521.

Bravard, J.P., Amoros, C. et Pautou, G., 1986. Impact of civil engineering works on the successions of communities in a fluvial system. Oikos, 47:92-111.

Bravard, J.P., Amoros, C., Pautou, G., Bornette, G., Boumaud, M., Châtelliers, M.C.D., Gilbert, J., Peiry, J.L., Perrin, J. et Tachet, H., 1997. River incision in southeast France: Morphological phenomena and ecological effects. Regulated Rivers: Research and Management, 13: 75-90.

Brierley, G.J., Cohen, T., Fryirs, K. et Brooks, A., 1999. Post-European changes to the fluvial geomorphology of Bega catchment, Australia : implications for river ecology. Freshwater Biology, $41:$ 839-848.

Brinson, M.M., Swift, B.L., Plantica, R.C. et Barclay, J.S., 1981. Riparian ecosystems : their ecology and status. United States Fish and Wildlife Service Biological Service Program, Washington D.C.

Carbiener, R., 1970. Un exemple de type forestier exceptionnel pour l'Europe occidentale: la forêt du lit majeur du Rhin au niveau du Fossé Rhénan (Fraxino-Ulmetum Oberd.53). Intérêt écologique et biogéographique. Comparaison avec d'autres forêts thermophiles. Vegetatio, $20: 97-148$.

Centre d'Études Techniques de l'Équipement de Lyon, 1982. Étude des variations morphologiques du lit de l'Allier. Ministère de I'Industrie, ClermontFerrand, $73 \mathrm{p}$.

Chevallier, H., 1996. Étude des forêts alluviales ligériennes-Typologie écologique et fonctionnelle, propositions de gestion. ENGREF, Nancy, 75 p.

Centre Régional de Documentation Pédagogique de Clermont-Ferrand, 1983. Histoire de la navigation sur l'Allier en bourbonnais. Documents Régionaux, Annales du Centre Régional de Documentation Pédagogique de ClermontFerrand, Clermont-Ferrand, $58 \mathrm{p}$.

Conservatoire des Espaces et Paysages d'Auvergne, 2001. Plan de gestion du Domaine Public Fluvial de l'Allier à Joze-Maringues (63) 2001-2005. Direction Départementale de l'Équipement du Puy-de-Dôme, ClermontFerrand, $95 \mathrm{p}$.

Cooper, D.J., Andersen, D.C. et Chimner, R.A., 2003. Multiple patchways for woody plant establishment on floodplains at a local to regional scales. Journal of Ecology, $91: 182-196$.

Cousins, S.A.O., 2001. Analysis of land-cover transitions based on 17th and 18 th century cadastral maps and aerial photographs. Landscape Ecology, $16: 41-54$.

Décamps, H., 1993. River margins and environmental change. Ecological Applications, 3: 441-445.

Décamps, H., Fortune, M., Gazelle, F. et Pautou, G., 1988. Historical influence of man on the riparian dynamics of fluvial landscape. Landscape Ecology, 1:163-173.

Dejaifve, P.A. et Pinoche, J.N., 1998. Plan de gestion de la Réserve Naturelle du Val d'Allier 1998-2002. Office National des Forêts, Moulins, 78 p. et annexes.

DIREN Auvergne-Epteau, 1998. Étude de l'Allier entre Vieille-Brioude et Villeneuve. Agence de l'eau Loire Bretagne, Orléans, $77 \mathrm{p}$.

Dufour, S., 2005. Contrôle naturels et anthropiques de la structure et la dynamique de la végétation des forêts riveraines. Exemples de différents hydro- 
systèmes rhodaniens. Thèse de doctorat, Université Jean Moulin Lyon III, $243 \mathrm{p}$.

Dufour, S. et Piégay, H., soumis. Changements fluviaux et forêts riveraines. In Actes du colloque Eaux et Forêts, Groupe d'Histoire des Forêts Françaises, septembre 2006, Bordeaux.

Eastman, J.R., 1997. Idrisi for Windows, User's guide. Worcester.

Eschner, T.R., Hadley, R.F. et Crowley, K.D., 1983. Hydrologic and morphologic changes in channels of the Platte River basin in Colorado, Wyoming and Nebraska: a historical perspective. United States Geological Survey, Reston, Professional Paper 1277A.

Fédération de la Région Auvergne pour la Nature et l'Environnement, 1982. Rivière Allier. Nature Vivante, Clermont-Ferrand, $110 \mathrm{p}$.

Gautier, E., 1992. Recherches sur la morphologie et la dynamique fluviales dans le bassin du Buèch (Alpes du Sud). Thèse de doctorat, Université de Paris X, $439 \mathrm{p}$.

Gautier, E., Piégay, H. et Bertaina, P., 2000. A methodological approach of fluvial dynamics oriented towards hydrosystem management: case study of the Loire and Allier rivers. Geodynamica Acta, 1: 29-43.

Gehrke, P.C. et Harris, J.H., 2001. Regional-scale effects of flow regulation on lowland riverine fish communities in new south Wales, Australia. Regulated Rivers: Research and Management, 17: 369-391.

Girel, J., 1986. Télédétection et cartographie à grande échelle de la végétation alluviale: exemple de la basse plaine de l'Ain, p. 45-74. In Recherches interdisciplinaires sur les écosystèmes de la Basse plaine de l'Ain (France). Université Scientifique, Technologique et Médicale de Grenoble, Grenoble.

Gregory, S.V., Swanson, F.J., McKee, W.A. et Cummins K.W., 1991. An ecosystem perspective of riparian zones. Bioscience, 41:540-551.

Grivel, S. et Gautier, E., 2000. Étude de l'évolution géomorphologique dynamique de la Loire à Bréhémont. Université d'Orléans, $130 \mathrm{p}$.

Gurnell, A.M. et Gregory, K.J., 1995. Interactions between semi-natural vegetation and hydrogeomorphological processes. Geomorphology, 13: 49-69.

Hughes, F.M.R., 1994. Environmental change, disturbance and regeneration in semi-arid floodplain forest, p. 321-345. In Biogeographical and Geomorphological Perspectives. John Wiley and Sons, Chichester.

Hughes, F.M.R., 1997. Floodplains biogeomorphology. Progress in Physical Geography, 21:501-529.

Hupp, C.R. et Osterkamp, W.R., 1985. Bottomland vegetation distribution along Passage Creek, Virginia, in relation to alluvial landforms. Ecology, 66:670681.

Johnson, W.C., 1994. Woodland expansion in the Platte River, Nebraska: patterns and causes. Ecological Monographs, 64:45-84.

Johnson, W.C., 1997. Equilibrium response of riparian vegetation to flow regulation in the Platte river, Nebraska. Regulated Rivers: Research and Management, 13: 403-415.

Johnson, W.C., Dixon M., Larson, G. et Simons, R., 1992. Riparian vegetation along the Snake river, Idaho, below Swan Falls Dam: past, present and future.

Junk, W.J., Bailey, P.B. et Sparks, R.E., 1989. The flood pulse concept in riverfloodplain systems. Canadian Journal of Fisheries and Aquatic Sciences, 106: 110-127.

Kingsford, R.T., 2000. Ecological impacts of dam, water diversions and river management on floodplain wetlands in Australia. Austra Ecology, 25: 109127.

Lelli, L., 2004. L'impact des politiques publiques agricoles sur les paysages en Nord-Comminges, p. 188-197. In Le défi du paysage, un projet pour l'agriculture. Les cahiers de la compagnie du paysage, Seyssel.

Lemaire, R., 1996. Comment le maïs s'est implanté dans le Val d'Allier. Revue Scientifique du Bourbonnais, 1994: 88-99.

Liébault, F. et Piégay, H., 2002. Causes of 20th century channel narrowing in mountain and piedmont rivers and stream of Southeastern France. Earth Surface Processes and Landforms, $27: 425-444$.
Luherne, P.L., 1994. Évolution des méandres en Val d'Allier bourbonnais. Conservatoire des Espaces et des Paysages d'Auvergne, Riom, 24 p.

Malanson, G., 1993. Riparian landscape. Cambridge University Press, Cambridge, $296 \mathrm{p}$.

Malavoi, J.-R., Bravard, J.-P., Piégay, H., Héroin, E. et Ramez, P., 1998. Détermination de l'espace de liberté des cours d'eau. Agence de l'eau Rhône Méditerranée Corse, Lyon, 39 p.

Marston, R.A., Girel, J., Pautou, G., Piégay, H., Bravard J.-P. et Arneson, C., 1995. Channel metamorphosis, floodplain disturbance and vegetation development: Ain river, France. Geomorphology, 13: 121-131.

Mendonça-Santos, M.L., 1999. GIS and spatiotemporal modelling for the study of alluvial soil and vegetation evolution. Thèse de Doctorat, École Polytechnique Fédérale de Lausanne, 142 p.

Mendonça-Santos, M.L. et Claramunt, C., 2001. An integrated landscape and local analysis of land cover evolution in an alluvial zone. Computers, Environment and Urban Systems, $25: 557-577$.

Merritt, D.M. et Cooper, D.J., 2000. Riparian vegetation and channel change in response to river regulation: a comparison study of regulated and unregulated streams in the Green River basin, USA. Regulated Rivers: Research and Management, $16: 543-564$.

Michelin, Y., 1995. Les jardins de Vulcain. Maison des Sciences de l'Homme, Paris, $155 \mathrm{p}$.

Miller, J.R., Schulz, T.T., Hobbs, N.T., Wilson, K.R., Schrupp, D.L. et Baker, W.L., 1995. Changes in the landscape structure of a southeastern Wyoming riparian zone following shifts in stream dynamics. Biological Conservation, 72 : 371-379.

Mondanel, P., 1975. L'ancienne batellerie de l'Allier et de la Dore (de Langeac à Nevers). De Bussac, Clermont-Ferrand, $689 \mathrm{p}$.

Naiman, R.J., 1996. Water, society and landscape ecology. Landscape Ecology, 11: 193-196.

Naiman, R.J., Décamps, H. et Pollock, M., 1993. The role of riparian corridors in maintaining biodiversity. Ecological Applications, 3: 209-212.

Nanson, G.C. et Knighton A.D., 1996. Anabranching rivers: their cause, character and classification. Earth Surface Processes and Landforms, $21: 217$ 239.

Pautou, G., 1984. L'organisation des forêts alluviales dans l'axe rhodanien entre Genève et Lyon, comparaison avec d'autres systèmes fluviaux, p. 43-64. In Document de Cartographie Écologique, Grenoble.

Pautou, G. et Décamps, H., 1985. Ecological interactions between the alluvial forests and hydrology of the Upper Rhône. Archiv fuer Hydrobiologie, 104 : 13-37.

Pautou, G., Girel, J. et Borel, J.-L., 1992. Initial repercussions and hydroelectric developments in the french Upper Rhône valley: a lesson for predictive scenarios propositions. Environmental Management, 16: 231-242.

Pautou, G., Ponsero, A. et Jouannaud, P., 1997. Les changements de biodiversité dans les interfaces alluviales. Application à la plaine d'inondation du Rhône entre Genève et Lyon et la Réserve Naturelle du marais de Lavours. Revue d'Écologie Alpine, IV : 35-63.

Peiry, J.L., Vivian, H., 1994. Dynamique des crues et réduction de la capacité du chenal consécutive à la construction d'un barrage hydroélectrique: l'exemple du Drac inférieur à l'amont de Grenoble, SHF. 23e journée de l'hydraulique: crues et inondations, Nîmes, 1:321-329.

Peiry, J.-L., 1997. Recherche en géomorphologie fluviale dans les hydrosystèmes fluviaux des Alpes du nord. Diplôme d'habilitation à diriger les recherches, Université Joseph Fourrier Grenoble I, Grenoble, 2 Vol., 307 p. et $341 \mathrm{p}$.

Piégay, H., 1995. Dynamique et gestion de la ripisylve de cinq cours d'eau à charge grossière du bassin du Rhône (l'Ain, l'Ardèche, le Giffre, l'Ouvèze et I'Ubaye), XIX-XX' siècles. Thèse de doctorat, Université Paris IV-Sorbonne, $529 \mathrm{p}$.

Piégay, H., 2000. Le bois mort en rivière faut-il toujours l'enlever? Comptes Rendus de l'Académie des Sciences, 86: 97-107. 
Piégay, H., Pautou, G. et Bravard, J.-P., 2003a. L'histoire contemporaine des marges fluviales: entre renaturation et dénaturation, p. 72-92. In Les forêts riveraines des cours d'eau, écologie, fonction et gestion. Institut pour le Développement Forestier, Paris.

Piégay, H., Peiry, J.-L. et Gazelle, F., 2003b. Effets des ripisylves sur la dynamique du lit et de son aquifère, p. 94-122. In Les forêts riveraines des cours d'eau, écologie, fonction et gestion. Institut pour le Développement Forestier, Paris

Piégay, H. et Schumm, S.A., 2003. System approaches in fluvial geomorphology, p. 105-134. In G.M. Kondolf et H. Piégay, édit., Tools in Fluvial Geomorphology. John Wiley and Sons, Chichester.

Pisùt, P., 2002. Channel evolution of the pre-channelized Danube river in Bratislava, Slovakia (1712-1886). Earth Surface Processes and Landforms, 27: 369-390.

Pontius, R.G., Shusas, E. et McEachern, M., 2004. Detecting important categorial land changes while accounting for persistence. Agriculture, Ecosystems and Environment, 101:215-268.

Puckridge, J.T., Sheldon, F., Walker, K.F. et Boulton, A.J., 1998. Flow variability and the ecology of large rivers. Marine and Freshwater Research, 49: 5572.

Rodrigues, S., 2004. Dynamique sédimentaire et végétation: évolution de chenaux fluviatiles en Loire moyenne (France). Thèse de doctorat, Université François Rabelais, $428 \mathrm{p}$.

Saillard, J., 1998. Étude de l'espace de liberté de l'Allier entre Joze et Maringues. Conservatoire des Espaces et des Paysages d'Auvergne, Riom, $52 \mathrm{p}$.

Saillard, J., 1999. Caractérisation géomorphologique et écologique de sites alluviaux sur la Loire et l'Allier. Conservatoire des Espaces et des Paysages d'Auvergne, Riom, $83 \mathrm{p}$.

Salo, J., Kalliola, R., Hakkinen, I., Makinen, Y., Neimela, P., Puhakka, M. et Coley, P., 1986. River dynamics and the diversity of Amazon lowland forest. Nature, 322: 254-258.
Schumm, S.A., 1977. The fluvial system. John Wiley and Sons, Chichester, $338 \mathrm{p}$.

Shankman, D., 1993. Channel migration and vegetation patterns in the southeastern coastal plain. Conservation Biology, 7: 176-183.

Sidle, R.C. et Sharma, A., 1986. Stream channel changes associated with mining and grazing in the Great Basin. Journal of Environmental Quality, 25: 1111-1121.

Steiger, J., Corenblit, D. et Vernier, P., 2000. Les ajustements morphologiques contemporains du lit mineur de la Garonne, France, et leurs effets sur l'hydrosystème fluvial. Zeitschrift für Geomorphologie, N.F. supplément 122 : 227-246.

Steiger, J., James, M. et Gazelle, F., 1998. Channelization and consequences on floodplain system functioning on the Garonne river, SW France. Regulated Rivers: Research and Management, 17: 443-460.

Steiger, J., Tabacchi, E., Dufour, S., Corenblit, D. et Peiry, J.-L., 2005. Hydrogeomorphic processes affecting riparian habitat within alluvial channel-floodplain river systems: A review for the temperate zone. River Research and Applications, $21: 719-737$.

Surian, N., 1999. Channel changes due to river regulation: the case of the Piave river, Italy. Earth Surface Processes and Landforms, 24 : 1135-1151.

Tal, M., Gran, K., Murray, A.B., Paola, C. et Hicks, D.M., 2004. Riparian vegetation as a primary control on channel characteristics in a multi-thread rivers. In S.J. Bennet et A. Simon, édit., Riparian Vegetation and Fluvial Geomorphology: Hydraulic, Hydrologic, and Geotechnical Interaction. American Geophysical Union, Washington.

Ward, J.V., Tockner, K. et Schiemer, F., 1999. Biodiversity of floodplain river ecosystems: ecotones and connectivity. Regulated Rivers: Research and Management, 15: 125-139.

Ward, J.V. et Wiens, J.A., 2001. Ecotones of riverine ecosystems: role and typology, spatiotemporal dynamics and river regulation. Ecohydrology and Hydrobiology, 1 : 25-36. 